This item was submitted to Loughborough's Research Repository by the author.

Items in Figshare are protected by copyright, with all rights reserved, unless otherwise indicated.

\title{
Association of gut-related metabolites with outcome in acute heart failure
}

PLEASE CITE THE PUBLISHED VERSION

https://doi.org/10.1016/j.ahj.2021.01.006

PUBLISHER

Elsevier

VERSION

AM (Accepted Manuscript)

PUBLISHER STATEMENT

This paper was accepted for publication in the journal American Heart Journal and the definitive published version is available at https://doi.org/10.1016/j.ahj.2021.01.006.

\section{LICENCE}

CC BY-NC-ND 4.0

\section{REPOSITORY RECORD}

Israr, Muhammad Zubair, Dennis Bernieh, Andrea Salzano, Shabana Cassambai, Yoshiyuki Yazaki, Liam Heaney, Donald JL Jones, Leong L Ng, and Toru Suzuki. 2021. "Association of Gut-related Metabolites with Outcome in Acute Heart Failure". Loughborough University. https://hdl.handle.net/2134/13606937.v1. 
Short title: Gut-related metabolites and outcomes in heart failure

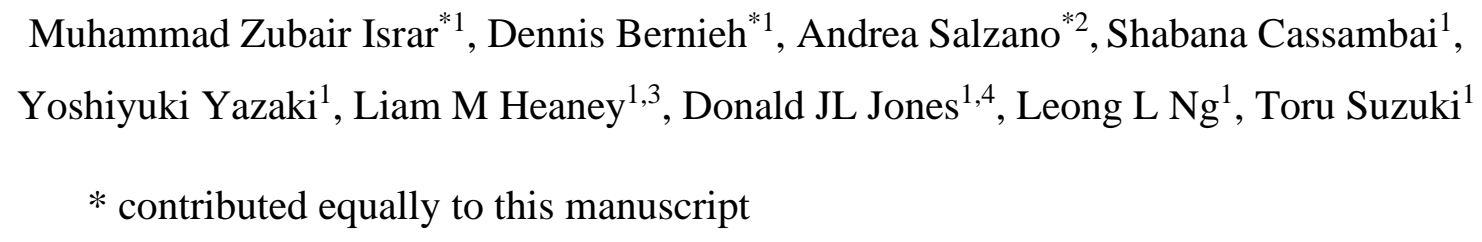

${ }^{3}$ School of Sport, Exercise \& Health Sciences, Loughborough University, Loughborough, LE11 3TU, UK

${ }^{4}$ Department of Cancer Studies, University of Leicester, RKCSB, Leicester, LE2 7LX, UK

\section{Address for Correspondence:}

Prof Toru Suzuki

Department of Cardiovascular Sciences and NIHR Leicester Cardiovascular Biomedical Research Centre, University of Leicester, Glenfield Hospital, Leicester, LE3 9QP, UK Email: ts263@le.ac.uk. Tel: (0044) 1162044741

Keywords: gut microbiota, heart failure, metabolomics, outcomes, carnitine, prognosis 


\section{$1 \quad$ ABSTRACT}

2 Background: Trimethylamine N-oxide (TMAO), a gut-related metabolite, is associated with

3 heart failure (HF) outcomes. However, TMAO is the final product of a complex metabolic

4 pathway (i.e. choline/carnitine) that has never been entirely investigated in HF. The present study investigates a panel of metabolites involved in the TMAO-choline/carnitine metabolic

6 pathway for their associations with outcome in acute HF patients.

7 Methods: In total, 806 plasma samples from acute HF patients were analysed for TMAO, trimethyllysine, L-carnitine, acetyl-L-carnitine, $\gamma$-butyrobetaine, crotonobetaine, trimethylamine, betaine aldehyde, choline, and betaine using a developed liquid chromatography-tandem mass spectrometry method. Associations with outcome of all-cause mortality (death) and a composite of all-cause mortality and/or rehospitalization due to HF (death/HF) at 30 days and 1 year were investigated.

Results: TMAO, trimethyllysine, L-carnitine, acetyl-L-carnitine, and $\gamma$-butyrobetaine were associated with death and death/HF at 30 days (short-term) (HR 1.30-1.49, $\mathrm{p} \leq 0.021)$ and at 1 year (long-term) $($ HR 1.15-1.25, $\mathrm{p} \leq 0.026)$ when adjusted for cardiac risk factors. L-carnitine and acetyl-L-carnitine were superior for short-term outcomes whereas TMAO was the superior metabolite for association with long-term outcomes. Furthermore, acetyl-L-carnitine and L-carnitine were superior for in-hospital mortality and improved risk stratification when combined with current clinical risk scores (i.e. ADHERE, OPTIMIZE-HF, GWTG-HF) $(\mathrm{OR} \geq 1.52, \mathrm{p} \leq 0.020)$

Conclusions: Carnitine-related metabolites show associations with adverse outcomes in acute HF, in particular L-carnitine and acetyl-L-carnitine for short-term outcomes, and TMAO for long-term outcomes. Further studies are warranted to investigate the role and implications of carnitine metabolites including intervention in the pathogenesis of HF. 
A growing body of evidence supports that the gut microbiota plays an important role in the pathogenesis of heart failure (HF) (1-3). In particular, trimethylamine N-oxide (TMAO), a gut-derived metabolite of the choline/carnitine metabolic pathway $(4,5)$, has been highlighted as a novel risk marker for outcomes of death in cardiovascular disease (6). Several studies have shown that elevated TMAO levels are associated with poor prognosis in both acute (6) and chronic HF patients (7-10). More recently, TMAO levels did not respond to guideline-based therapy in a large multicentre HF cohort despite being associated with death due to HF $(11,12)$. Furthermore, the combination of TMAO to the current goldstandard HF biomarker, B-type natriuretic peptide (BNP), showed improvements in risk (13). carnitine, L-carnitine, $\gamma$-butyrobetaine, and TML, individually and in combination for their 
METHODS

\section{Study population}

Eight hundred and six patients, with available plasma sample volume, admitted with acute HF to the University Hospitals of Leicester, UK between February 2006 and August 2011 were enrolled, as previously described $(6,16)$. Each patient consented to have blood samples taken and outcomes surveyed. This study was approved by the local ethics committee and complied with the Declaration of Helsinki. Diagnosis of acute HF was made according to clinical signs and symptoms, following ESC guidelines (17). Exclusion criteria included patients with previous history of cancer or renal replacement therapy, surgical procedure within the previous month, presence of cardiogenic shock, sepsis, pneumonia, or acute coronary syndromes, and inability to consent (e.g. dementia).

The primary endpoints were all-cause mortality (death) and a composite of death and/or rehospitalization due to $\mathrm{HF}$ (death/HF) at 30 days and at 1 year. Where multiple events occurred, the time to the first event was counted as the outcome. Determinations of inclusion and endpoints were performed by an independent cardiologist.

\section{Sample collection and measurement of biomarkers}

Peripheral intravenous blood samples were obtained within the $24 \mathrm{~h}$ period after admission to hospital with acute HF and collected in pre-chilled tubes containing EDTA and aprotinin. Plasma was separated via centrifugation at $1500 \mathrm{~g}$ for $20 \mathrm{~min}$ at $4^{\circ} \mathrm{C}$ and stored in aliquots at $-80^{\circ} \mathrm{C}$ until analysis. For analysis, samples were thawed at room temperature and analyzed immediately following preparation.

N-terminal pro B-type natriuretic peptide (NT-proBNP) was measured in all patients using a sandwich immunoassay as described previously (18). 
choline, L-carnitine, betaine, betaine aldehyde and crotonobetaine were extracted from $50 \mu \mathrm{l}$ of plasma using stable-isotope dilution and derivatization. Extracted samples were analysed by UHPLC-MS/MS with multiple reaction monitoring (MRM) using a Shimadzu UPLC Nexera X2 LC30AD with an Acquity UPLC BEH HILIC $100 \mathrm{~mm}$ analytical column coupled to a Shimadzu 8050 triple quadrupole mass spectrometer (Shimadzu Limited, Kyoto Japan). Detailed description of the methods is described in the Supplementary Material.

\section{Statistical analyses}

Spearman correlations were calculated for the metabolites and clinical parameters.

Cox proportional hazards regression analyses were performed to identify associations with death/HF at 30 days and 1 year. Metabolite levels were log transformed, and normalised to 1standard deviation, so that hazard ratios refer to Z-transformed variables. Kaplan-Meier survival curves were generated to show the relationship between the metabolites and the outcomes of death/HF. Mantel-Cox log rank tests were used to report the significance of stratification by tertiles for the metabolites. To analyse the predictive power of the metabolites for in-hospital mortality, binary logistic regression analyses were performed and included the Acute Decompensated HEart Failure National REgistry (ADHERE), Organized Program To Initiate Lifesaving Treatment In Hospitalized Patients With Heart Failure (OPTIMIZE-HF), and (Get With The Guidelines-Heart Failure) GWTG-HF scores (19-21). The ADHERE risk score was categorised against the first group (reference) and patients were assigned to risk groups using the ADHERE registry model. Outcome prediction accuracies were assessed by calculating the area under the curve (AUC) for the receiver operator characteristics (ROC) curve analysis and reclassification analyses were used to assess the clinical utility of adding individual metabolites and combination of metabolites to a basic model of clinical cardiovascular risk. A p-value of $<0.05$ was deemed to be statistically 
1 significant. Statistical analyses were performed using IBM SPSS Statistics (V26, IBM Corp.,

2 Armonk, New York, USA).

3

4

5

6

7

8

9

10

11

12

13

14

15

16

17

18

19

20 
RESULTS

\section{Patient demographics}

Eight hundred and six patients, admitted with acute HF, were investigated for the described metabolites. The majority of patients were of NYHA class III/IV (95\%), male (61\%) with a median age of 78 years. A total of 313 events were recorded during the 1-year follow-up period with 57 deaths during in-hospital care, 154 deaths post-discharge and 100 readmissions to hospital due to HF. Clinical demographics and measured endpoints are shown in Table 1.

\section{Associations with clinical parameters}

Spearman's correlation analysis was performed to assess the correlations between the metabolites and clinical parameters. Of the metabolites, acetyl-L-carnitine, betaine, choline, $\gamma$-butyrobetaine, L-carnitine, TMA, TMAO and TML were quantifiable in our cohort and showed positive correlations with each other $\left(\mathrm{r}_{\mathrm{s}}=0.099-0.737, \mathrm{p} \leq 0.005\right)$ and negative correlations with systolic blood pressure $\left(r_{s}=-0.103--0.167, p \leq 0.005\right)$. Apart from betaine, all other metabolites showed correlations with renal markers, eGFR $\left(r_{s}=-0.123--0.437\right.$, $\mathrm{p} \leq 0.001)$, and urea $\left(\mathrm{r}_{\mathrm{s}}=0.142-0.392, \mathrm{p}<0.001\right)$. Correlations with available clinical parameters are detailed in Supplementary Table 6.

The association between metabolites and clinical characteristics was also investigated. Only TMA showed differences in distribution across severity for NYHA class III and IV patients (mean rank $350.6 \mathrm{pmol} / \mathrm{L}$ and $401.8 \mathrm{pmol} / \mathrm{L}$ respectively, $\mathrm{p}=0.001$ ), whereas betaine alone showed differences based on ejection fraction $(<40 \%$ and $\geq 40 \%$, mean rank 323.3 $\mathrm{pmol} / \mathrm{L}$ and $265.69 \mathrm{pmol} / \mathrm{L}$ respectively, $\mathrm{p}<0.001$ ). For distribution with treatment, apart from betaine, all other metabolites showed lower levels in patients who were given beta-blockers $(p \leq 0.036)$, with only TML showing differences for angiotensin-converting enzyme (ACE) 
inhibitor or angiotensin receptor blocker (ARBs), with patients given ACE/ARBs reporting higher levels of TML ( $\mathrm{p}=0.034)$.

\section{Associations with death/HF}

Cox survival analyses were conducted to investigate the association of independent metabolites with death/HF at 30 days and 1 year. Models were adjusted for sex, age, previous medical history (HF, ischaemic heart disease, hypertension, and diabetes mellitus), New York Heart Association (NYHA) class, smoking status, oedema, atrial fibrillation, systolic blood pressure, diastolic blood pressure, heart rate, haemoglobin, respiratory rate, blood sodium and ( $\log$ ) NT-proBNP. Of the metabolites analysed, univariate analysis showed that TMAO, TML, L-carnitine, $\gamma$-butyrobetaine, and acetyl-L-carnitine were all associated with death/HF across both timepoints [HR 1.20-1.79 (95\% CI 1.07-2.16) $\mathrm{p} \leq 0.002]$. After adjustment, acetylL-carnitine, $\gamma$-butyrobetaine, L-carnitine, TMAO and TML showed independent associations at 30 days [HR 1.30-1.49 (95\% CI 1.04-1.88) p $\leq 0.021$ ] and 1 year [HR 1.15-1.25 (95\% CI 1.02-1.42) $\mathrm{p} \leq 0.026]$ (Table 2). Betaine, choline, and TMA did not show independent associations with outcome across either timepoint as univariate or multivariate markers.

For short-term outcome at 30 days, elevated acetyl-L-carnitine levels were associated with an increase in death/HF [HR $1.49(95 \%$ CI 1.19-1.88) $\mathrm{p}=0.001)]$, whereas for long-term outcomes TMAO was associated with an increase in death/HF after adjustment [HR 1.25 (95\% CI 1.09-1.42) $\mathrm{p}=0.001]$.

Additionally, after the inclusion of renal markers (urea and eGFR), none of the metabolites showed significant associations with the outcome of death/HF across all endpoints, indicating a strong confounding influence from biomarkers of renal function (Supplementary Table 7). Furthermore, Cox survival analysis were performed by splitting the population into ischaemic HF and non-ischaemic HF. Results showed that in patients with 
1 ischaemic HF, none of the metabolites were significant across both timepoints. However, in

2 patients with non-ischaemic HF, only TMAO was significant at both timepoints [HR 1.24-

3

4

5

6

7

8

9

10

11

12 1.48 (95\% CI 1.04-1.95) $\mathrm{p} \leq 0.017]$ (Supplementary Table 8).

For Kaplan-Meier survival analysis, patients were stratified based on tertile levels of each metabolite and analysed for association with events of death/HF. Results showed that TMAO, TML, L-carnitine, acetyl-L-carnitine, and $\gamma$-butyrobetaine showed association of reduced survival with elevated levels at 1 year $(\mathrm{p} \leq 0.005)$ (Supplementary Figure 2$)$. Receiver operating characteristics (ROC) analysis showed incremental decreases over time from 30 days to 1 year for acetyl-L-carnitine, $\gamma$-butyrobetaine, L-carnitine, TMAO and TML [AUC $(95 \% \mathrm{CI})$ at 30 days were $0.606-0.675(0.548-0.730) \mathrm{p}<0.001$; and at 1 year were $0.563-0.602$ $(0.523-0.643) \mathrm{p} \leq 0.002]$. Betaine, choline and TMA were not significant across any timepoint ( $\mathrm{p} \geq 0.073$ ) (Table 3).

\section{Associations with mortality}

Cox survival analyses were performed for death and adjusted using the same aforementioned model. Univariate analysis showed that TMAO, TML, L-carnitine, $\gamma$ butyrobetaine, and acetyl-L-carnitine were associated with death at 30 days [HR 1.43-2.24 (95\% CI 1.11-2.81) $\mathrm{p} \leq 0.005]$, and betaine, TMA, TMAO, TML, L-carnitine, $\gamma$-butyrobetaine, and acetyl-L-carnitine at 1 year [HR $1.17-1.50(95 \%$ CI $1.03-1.50) \mathrm{p} \leq 0.018]$. When adjusted, betaine, choline, and TMA were not associated with mortality at 30 days nor at 1 year. The remaining metabolites showed associations with death at 30 days [HR 1.39-1.81 (95\% CI $1.05-2.42) \mathrm{p} \leq 0.022]$ and at 1 year [HR 1.26-1.34 (95\% CI 1.08-1.58) $\mathrm{p} \leq 0.004]$ after adjustment (Table 2). 

was associated with outcome at 30 days [HR 1.37 (95\% CI 1.01-1.85) $\mathrm{p}=0.045$ ], as was $\gamma$ butyrobetaine at 1 year [HR 1.19 (95\% CI 1.01-1.40) p=0.042] (Supplementary Table 7).

Kaplan-Meier survival analysis for death when stratified by tertiles showed association of reduced survival with elevated levels of acetyl-L-carnitine, $\gamma$-butyrobetaine, Lcarnitine, TMA, TMAO and TML at 1 year $(\mathrm{p} \leq 0.003)$ (Supplementary Figure 3 ).

\section{Associations with in-hospital mortality}

For ROC analysis, acetyl-L-carnitine reported the highest AUC for association with in-hospital mortality [AUC 0.744 (95\% CI 0.67-0.82) $\mathrm{p}<0.001$ ]. Betaine, $\gamma$-butyrobetaine, Lcarnitine, TMA, TMAO and TML also showed associations with in-hospital mortality [AUC 0.586-0.684 (95\% CI 0.513-0.758) $\mathrm{p} \leq 0.022]$ (Table 3).

Analyses were performed to evaluate the added value of the metabolites with clinical risk scores as either a categorical (ADHERE) or continuous variable (ADHERE, OPTIMIZEHF and GWTG-HF). The levels of each metabolite showed differences when stratified by the ADHERE categorical score $(\mathrm{p} \leq 0.002)$. Pairwise comparison against the reference group (ADHERE group 1) showed significant differences and elevations in groups 3-5 for TMAO (adj. $\mathrm{p} \leq 0.008$ ), and group 3 and 4 for acetyl-L-carnitine, choline, $\gamma$-butyrobetaine, $\mathrm{L}$ carnitine, TMA, and TML (adj. $\mathrm{p} \leq 0.035$ ) (Figure 2). When the metabolites were added to the base model of the clinical risk scores, only acetyl-L-carnitine, L-carnitine and $\gamma$-butyrobetaine $(p \leq 0.020)$ were associated with in-hospital mortality for all three clinical risk scores, with a strong influence from renal function biomarkers, eGFR, and urea (Figure 3). 
Net reclassification analyses were performed by adding each metabolite to each of the three scoring systems (ADHERE, OPTIMIZE-HF and GWTG-HF). The results showed that in the present cohort, only acetyl-L-carnitine, L-carnitine, $\gamma$-butyrobetaine, and TMA showed overall improvements in reclassification when added to the ADHERE score $(p \leq 0.037)$.

Furthermore, when added to the OPTIMIZE-HF score, improvements were seen with the addition of choline, acetyl-L-carnitine, L-carnitine, and $\gamma$-butyrobetaine $(\mathrm{p} \leq 0.035)$. Overall improvements were also seen with betaine, acetyl-L-carnitine, $\gamma$-butyrobetaine, and Lcarnitine when added to the GWTG-HF score $(\mathrm{p} \leq 0.007)$ (Table 4).

\section{Associations of combined metabolites with outcomes}

As TMA, choline, and betaine did not show associations as univariate or multivariate markers with death/HF, they were discounted from combinatorial statistical analysis.

Logistic regression analysis for associations with death/HF at 30 days (short-term) and 1 year (long-term) identified TMAO and acetyl-L-carnitine as prospective markers in a combinatorial model. Furthermore, these metabolites were also confirmed as those with the highest area under the curve (AUC) on ROC curve analysis (Table 3). When combined, the ROC curve analysis did not show additional advantages for combined metabolites at shortterm ( $\mathrm{p} \geq 0.086)$ nor long-term ( $\mathrm{p}=0.934)$ outcomes of death/HF for continuous analysis.

For binary analysis, the Youden's Index for optimal cut off point from the ROC curve for death/HF at 1 year was applied (TMAO $16.7 \mu \mathrm{mol} / \mathrm{L}$ and acetyl-L-carnitine $18.5 \mu \mathrm{mol} / \mathrm{L}$ ), with both metabolites yielding approximately identical categories $(71 \%$ of patients below the cut-off for TMAO and 74\% for acetyl-L-carnitine) $(22,23)$. Kaplan-Meier survival curve analysis was performed for death/HF at 30 days and at 1 year using the same cut-off point. Results showed that TMAO and acetyl-L-carnitine showed association of reduced survival 
1 with elevated levels at 30 days $(\mathrm{p}<0.001)$ and at 1 year $(\mathrm{p}<0.001)$. The metabolites were

2 combined as a risk score of four categories (both below-cut off, TMAO above-cut off and

3 acetyl-L-carnitine below-cut off, TMAO-below cut off and acetyl-L-carnitine above-cut off,

4 and both above-cut off). Results for death/HF at 30 days showed that elevated levels of both

5 metabolites showed reduced survival, superior to elevations of either metabolite or no

6 metabolite elevated ( $\mathrm{p} \leq 0.033$, chi-square $\geq 4.526)$. Similarly, for death/HF at 1 year, results

7 showed reduced survival when both metabolites were elevated when compared to other

8 groupings $(p \leq 0.022$, chi-square $\geq 5.281)$ (Supplementary Figure 4$)$. Interestingly, for short-

9 term outcomes, low levels of both metabolites and high TMAO-low acetyl-L-carnitine

10 showed no significant difference $(\mathrm{p}=0.212)$ whereas at long-term outcomes, low levels of

11 both metabolites and low TMAO-high acetyl-L-carnitine showed no significant difference ( $\mathrm{p}=0.062$ ), suggesting that acetyl-L-carnitine is the weighing factor for short-term outcomes and TMAO for long-term. 
The present study analysed a panel of TMAO-choline/carnitine pathway metabolites, with several novel findings. Firstly, carnitine-related TMAO precursors (trimethyllysine, Lcarnitine, acetyl-L-carnitine, and $\gamma$-butyrobetaine) showed association with HF outcomes [death and death/HF at 30 days (short-term) and at 1 year (long-term)], whilst choline-derived metabolites (choline and betaine) were not associated with outcomes. Secondly, metabolites showed different association with outcomes; specifically, acetyl-L-carnitine and L-carnitine both showed superiority over the other metabolites for short-term associations and in-hospital mortality in combination with current clinical risk scoring methods, whilst TMAO was not only associated with outcome, as previously reported, but was also the most performant for long-term outcomes, suggesting that the different metabolites are useful for risk stratification at different stages (short-/long-term outcomes).

Previous studies have identified the gut-derived metabolite, TMAO, and associated metabolites, TML and $\gamma$-butyrobetaine, to show associations with outcomes of HF and other cardiovascular diseases (CVD) (6-10,24). Mechanistically, TMAO is derived from two principal pathways that involve choline and/or carnitine $(1,4,5)$. Metabolites of the choline pathway, such as choline and its downstream betaine, showed elevated circulating levels in patients with chronic HF and correlations with clinical characteristics (e.g. haemodynamic status, disease severity); however, as in our report, associations with outcomes of death/HF during follow-up have not been reported (25). In contrast, metabolites associated with the carnitine pathway (L-carnitine, acetyl-L-carnitine, $\gamma$-butyrobetaine, and trimethyllysine) have shown associations with adverse outcomes of death/HF. Specifically, trimethyllysine has been reported to show associations with short- and long-term cardiovascular events (15), and its derivative, $\gamma$-butyrobetaine, has been identified to be a CVD risk marker (14). 
2 Firstly, L-carnitine has an essential role in fatty acid metabolism and carbohydrate metabolism via acetyl-L-carnitine. Mechanistically, within the mitochondrial matrix, fatty acid oxidation is dependent on esterification of fatty acyl coA esters together with acylcarnitines to allow transport into the mitochondria (26). Under physiological conditions, in the normal heart ATP is generated from mitochondrial oxidative phosphorylation by glucose and fatty acid oxidation, with the former as the most energy efficient. By contrast, in the pathological heart of HF, there is a shift in cardiac energy metabolism associated with decreased mitochondrial oxidative phosphorylation and chronic activation of the reninangiotensin-aldosterone and adrenergic systems, resulting in a shift in energy metabolism and an overall net reduction in energy efficiency (27). In HF, when left ventricular dysfunction occurs, there is a subsequent reduction in myocardial intra-cellular carnitine levels (28), which is associated with a shift from glucose to fatty acid oxidation as the main source of oxidative metabolism with an increased production of reactive oxygen species, that contribute to cardiac damage $(29,30)$, together with the reduction of the phosphocreatine/ATP ratio which determines cardiac energy loss and dysfunction (31). In this context, increased circulating carnitine levels have been proposed to represent a compensatory mechanism in $\mathrm{HF}$, in the attempt of improving fatty acid transport for metabolism and oxidation, thereby improving myocardial function (32). In line with this concept, our observation that circulating levels of L-carnitine and acetyl-L-carnitine are the most performant for short-term outcomes, suggests that their measurement may identify patients with a more severe impairment of cardiac bioenergetics which results in outcomes of death/HF.

Collectively, our results suggest that the TMAO-choline/carnitine pathway is affected differently during phases of HF; for instance, patients with a more impaired mitochondrial function have increased L-carnitine levels with a very poor outcome, whereas patients with 
1 less impaired mitochondrial bioenergetics and a longer survival show increased levels of

2 TMAO, which were most associated with poor outcome in the long term. Notably, in our cohort, apart from betaine, all metabolites showed lower levels in patients who were given beta-blockers, suggesting the effects of adrenergic modulation on the metabolic pathway. Further, L-carnitine has also been implicated in muscle wasting conditions (i.e. sarcopenia), via the same mechanism discussed above and demonstrated in skeletal wasting/cardiac cachexia, a typical feature of advanced HF $(33,34)$. In addition, carnitine insufficiency is common amongst HF patients, and has been associated with reduced left ventricular diastolic function. Carnitine supplementation has been reported as a possible treatment of mitochondrial dysfunction in $\operatorname{HF}(35,36)$, leading to improvement in clinical symptoms, cardiac morphology and function, natriuretic peptides, and renal function; however, no clear effects on mortality have been demonstrated $(37,38)$. These beneficial findings are linked both to the metabolic effect on myocardial cells (39), through an increase in glucose utilisation rather than a normalisation of the fatty acid metabolism (39), and to the anticatabolic effect on skeletal muscle cells, resulting in the L-carnitine anti-wasting effect (40). Our results, showing that patients with higher levels of L-carnitine and acetyl-L-carnitine (i.e. patients with a more severe impairment of mitochondrial bioenergetics) have the poorest short-term prognosis, further support the important role of the carnitine pathway in HF, suggesting that further studies on the role of carnitine are needed.

\section{Study Limitations}

As study limitations, patients were recruited from a single centre with the majority being Caucasians, and data regarding dietary intake that could influence metabolite levels were not available. Furthermore, crotonobetaine and betaine aldehyde were below the limit of quantitation for this method. Considering that these metabolites were quantified in previous studies at very low concentrations (41), it is reasonable to hypothesise that the reportedly low 
1 concentrations as well as the lack of quantification in our cohort are due to these metabolites

2 being transient intermediates. This method was not developed to detect acyl-L-carnitine and

3 therefore acetyl-CoA (product of beta-oxidation) and acetyl-L-carnitine:free L-carnitine ratio

4 was not reported in the present investigation.

5

6 CONCLUSIONS

7 In conclusion, the present study demonstrates the role of metabolites of the TMAO-

8 carnitine/choline metabolic pathway in risk stratification of acute HF, with a preponderant

9 role played by carnitine-related metabolites. In addition to risk stratification, carnitine

10 intervention may potentially have a therapeutic role which needs to be further investigated.

11

12

13

14

15

16

17

18

19

20

21 


\section{Conflicts of interest}

2 The authors report no conflict of interest.

3

\section{$4 \quad$ Funding}

5 This work was supported by the Japan Heart Foundation, National Institute for Health

6 Research (Leicester Biomedical Research Centre), the British Heart Foundation (BHF) and

7 the Medical Research Council (MRC) UK Consortium on MetAbolic Phenotyping

8 (MAP/UK) to TS.

\section{Acknowledgements}

10 Dr Salzano receives research grant support from CardioPath, Department of Advanced

11 Biomedical Sciences, Federico II University, Naples, Italy, and UniNA and Compagnia di

12 San Paolo in the frame of the STAR (Sostegno Teritoriale alla Attività di Ricerca)

13 programme.

14

15

16

17 
2 1. Salzano A, Cassambai S, Yazaki Y et al. The Gut Axis Involvement in Heart Failure: Focus on Trimethylamine N-oxide. Heart Fail Clin 2020;16:23-31.

4 2. Busnelli M, Manzini S, Chiesa G. The Gut Microbiota Affects Host Pathophysiology as an Endocrine Organ: A Focus on Cardiovascular Disease. Nutrients 2020;12:79.

3. Tang WW, Li DY, Hazen SL. Dietary metabolism, the gut microbiome, and heart failure. Nat Rev Cardiol 2019;16:137-154.

4. Cassambai S, Salzano A, Yazaki Y et al. Impact of acute choline loading on

6. Suzuki T, Heaney LM, Bhandari SS, Jones DJ, Ng LL. Trimethylamine N-oxide and prognosis in acute heart failure. Heart 2016;102:841-848.

7. Tang WW, Wang Z, Fan Y et al. Prognostic value of elevated levels of intestinal microbe-generated metabolite trimethylamine-N-oxide in patients with heart failure: refining the gut hypothesis. J Am Coll Cardiol 2014;64:1908-1914.

8. Trøseid M, Ueland T, Hov J et al. Microbiota-dependent metabolite trimethylamine$\mathrm{N}$-oxide is associated with disease severity and survival of patients with chronic heart failure. J Intern Med 2015;277:717-726.

9. Schuett K, Kleber ME, Scharnagl H et al. Trimethylamine-N-oxide and heart failure with reduced versus preserved ejection fraction. J Am Coll Cardiol 2017;70:32023204. 
1 10. Hayashi $\mathrm{T}$, Yamashita $\mathrm{T}$, Watanabe $\mathrm{H}$ et al. Gut microbiome and plasma microbiomerelated metabolites in patients with decompensated and compensated heart failure. Circ J 2018;83:182-192.

11. Suzuki T, Yazaki Y, Voors AA et al. Association with outcomes and response to treatment of trimethylamine N-oxide in heart failure: results from BIOSTAT-CHF. Eur J Heart Fail 2019;21:877-886.

12. Yazaki Y, Salzano A, Nelson CP et al. Geographical location affects the levels and association of trimethylamine $\mathrm{N}$-oxide with heart failure mortality in BIOSTAT-CHF: a post-hoc analysis. Eur J Heart Fail 2019.

13. Salzano A, Israr MZ, Yazaki Y et al. Combined use of trimethylamine N-oxide with BNP for risk stratification in heart failure with preserved ejection fraction: findings from the DIAMONDHFpEF study. Eur J Prev Cardiol 2019:2047487319870355.

14. Skagen K, Trøseid M, Ueland T et al. The Carnitine-butyrobetaine-trimethylamine-Noxide pathway and its association with cardiovascular mortality in patients with carotid atherosclerosis. Atherosclerosis 2016;247:64-69.

15. Li XS, Obeid S, Wang Z et al. Trimethyllysine, a trimethylamine N-oxide precursor, provides near-and long-term prognostic value in patients presenting with acute coronary syndromes. Eur Heart J 2019;40:2700-2709.

16. Suzuki T, Israr MZ, Heaney LM, Takaoka M, Squire IB, Ng LL. Prognostic role of molecular forms of B-type natriuretic peptide in acute heart failure. Clin Chem 2017;63:880-886.

17. McMurray JJV, Adamopoulos S, Anker SD et al. ESC Guidelines for the diagnosis and treatment of acute and chronic heart failure 2012. Eur J Heart Fail 2012;14:803869. 
1 18. Omland $\mathrm{T}$, Persson $\mathrm{A}, \mathrm{Ng} \mathrm{L}$ et al. N-terminal pro-B-type natriuretic peptide and longterm mortality in acute coronary syndromes. Circulation 2002;106:2913-2918.

19. Abraham WT, Fonarow GC, Albert NM et al. Predictors of in-hospital mortality in patients hospitalized for heart failure: insights from the Organized Program to Initiate Lifesaving Treatment in Hospitalized Patients with Heart Failure (OPTIMIZE-HF). J Am Coll Cardiol 2008;52:347-356.

20. Fonarow GC, Adams KF, Abraham WT, Yancy CW, Boscardin WJ, Committee ASA. Risk stratification for in-hospital mortality in acutely decompensated heart failure: classification and regression tree analysis. JAMA 2005;293:572-580.

21. Peterson PN, Rumsfeld JS, Liang L et al. A validated risk score for in-hospital mortality in patients with heart failure from the American Heart Association get with the guidelines program. Circ Cardiovasc Qual Outcomes 2010;3:25-32.

22. Youden WJ. Index for rating diagnostic tests. Cancer 1950;3:32-35.

23. Salzano A, Israr MZ, Garcia DF et al. Circulating cell-free DNA levels are associated with adverse outcomes in heart failure: testing liquid biopsy in heart failure. Eur $\mathbf{J}$ Prev Cardiol 2020:2047487320912375.

24. Suzuki T, Heaney LM, Jones DJ, Ng LL. Trimethylamine N-oxide and risk stratification after acute myocardial infarction. Clin Chem 2017;63:420-428.

25. Meyer KA, Shea JW. Dietary choline and betaine and risk of CVD: a systematic review and meta-analysis of prospective studies. Nutrients 2017;9:711.

26. Ussher JR, Lopaschuk GD, Arduini A. Gut microbiota metabolism of L-carnitine and cardiovascular risk. Atherosclerosis 2013;231:456-461.

27. Katz AM. Cellular mechanisms in congestive heart failure. Am J Cardiol 1988;62:3A8A. 
1 28. Wang ZY, Liu YY, Liu GH, Lu HB, Mao CY. 1-Carnitine and heart disease. Life Sci 2018;194:88-97.

29. Ohira H, deKemp R, Pena E et al. Shifts in myocardial fatty acid and glucose metabolism in pulmonary arterial hypertension: a potential mechanism for a maladaptive right ventricular response. Eur J Echocardiogr 2015;17:1424-1431.

30. Taylor M, Wallhaus TR, DeGrado TR et al. An evaluation of myocardial fatty acid and glucose uptake using PET with [18F] fluoro-6-thia-heptadecanoic acid and [18F] FDG in patients with congestive heart failure. J Nucl Med 2001;42:55-62.

31. Lemieux H, Semsroth S, Antretter H, Höfer D, Gnaiger E. Mitochondrial respiratory control and early defects of oxidative phosphorylation in the failing human heart. Int $\mathbf{J}$ Biochem Cell Biol 2011;43:1729-38.

32. Ueland T, Svardal A, Øie E et al. Disturbed carnitine regulation in chronic heart failure - increased plasma levels of palmitoyl-carnitine are associated with poor prognosis. Int J Cardiol 2013;167:1892-1899.

33. Valentova M, Anker SD, von Haehling S. Cardiac Cachexia Revisited: The Role of Wasting in Heart Failure. Heart Fail Clin 2020;16:61-69.

34. Rosca MG, Hoppel CL. Mitochondrial dysfunction in heart failure. Heart Fail Rev 2013;18:607-22.

35. Bauer J, Morley JE, Schols AMWJ et al. Sarcopenia: A Time for Action. An SCWD Position Paper. J Cachexia Sarcopenia Muscle 2019;10:956-961.

36. Brown DA, Perry JB, Allen ME et al. Expert consensus document: Mitochondrial function as a therapeutic target in heart failure. Nat Rev Cardiol 2017;14:238-250.

37. Song X, Qu H, Yang Z, Rong J, Cai W, Zhou H. Efficacy and Safety of L-Carnitine Treatment for Chronic Heart Failure: A Meta-Analysis of Randomized Controlled Trials. Biomed Res Int 2017;2017:6274854. 
1 38. Kinugasa Y, Sugihara S, Yamada K et al. Carnitine insufficiency is associated with adverse outcomes in patients with heart failure with preserved ejection fraction. $\mathbf{J}$ Aging Res Clin Pract inpress Published Online First 2016;22.

39. Broderick TL, Panagakis G, DiDomenico D et al. L-carnitine improvement of cardiac function is associated with a stimulation in glucose but not fatty acid metabolism in carnitine-deficient hearts. Cardiovasc Res 1995;30:815-820.

40. Ringseis R, Keller J, Eder K. Mechanisms underlying the anti-wasting effect of Lcarnitine supplementation under pathologic conditions: evidence from experimental and clinical studies. Eur J Nutr 2013;52:1421-42.

41. Koeth RA, Levison BS, Culley MK et al. $\gamma$-Butyrobetaine is a proatherogenic intermediate in gut microbial metabolism of L-carnitine to TMAO. Cell Metab 2014;20:799-812. 


\section{TABLES}

2 Table 1 Patient demographics for acute HF patients at the time of admission to hospital.

\begin{tabular}{|c|c|}
\hline Age (yrs) & $78(69-84)$ \\
\hline Male & $61 \%$ \\
\hline Systolic BP (mmHg) & $133(115-150)$ \\
\hline Diastolic BP (mmHg) & $75(65-85)$ \\
\hline Heart rate (beats/min) & $90(74-107)$ \\
\hline de novo HF & $32 \%$ \\
\hline Past history IHD & $31 \%$ \\
\hline Past history diabetes & $34 \%$ \\
\hline Past history HTN & $58 \%$ \\
\hline Past history COPD & $9 \%$ \\
\hline Past history hyperlipidemia & $24 \%$ \\
\hline Current smoker & $10 \%$ \\
\hline Orthopnea & $52 \%$ \\
\hline Oedema & $60 \%$ \\
\hline Raised JVP & $56 \%$ \\
\hline Pulmonary oedema & $32 \%$ \\
\hline HFrEF $(\%) *$ & $58 \%$ \\
\hline Beta-blocker (\%) & $41 \%$ \\
\hline ACE/ARBs (\%) & $54 \%$ \\
\hline NYHA Class IV & $53 \%$ \\
\hline III & $42 \%$ \\
\hline & $\begin{array}{r}5 \% \\
<1 \%\end{array}$ \\
\hline Atrial fibrillation & $46 \%$ \\
\hline Respiratory rate (breaths/min) & $22(18-26)$ \\
\hline Urea $(\mathbf{m m o l} / \mathbf{L})$ & $8.9(6.6-12.8)$ \\
\hline Creatinine $(\mu \mathrm{mol} / \mathrm{L})$ & $112(91-141)$ \\
\hline eGFR $\left(\mathrm{mL} / \mathrm{min} / 1.73 \mathrm{~m}^{2}\right)$ & $52(39-67)$ \\
\hline $\mathrm{Na}^{+}(\mathbf{m m o l} / \mathbf{L})$ & $138(135-141)$ \\
\hline $\mathbf{K}^{+}(\mathbf{m m o l} / \mathbf{L})$ & $4.4(4.0-4.7)$ \\
\hline Hemoglobin $(\mathrm{g} / \mathrm{L})$ & $123(108-137)$ \\
\hline NT-proBNP (pmol/L) & $2160(1018-3726)$ \\
\hline ADHERE group 3-5 & $17 \%$ \\
\hline
\end{tabular}


OPTIMIZE-HF score

GWTG-HF score

Metabolites ( $\mu \mathrm{mol} / \mathrm{L})$

Acetyl-L-carnitine

$12.1(8.1-19.4)$

Betaine

45.7 (33.0-67.1)

Choline

$\gamma$-butyrobetaine

$0.51(0.86-1.49)$

L-carnitine

$52.1(39.2-77.4)$

Trimethylamine

$0.27(0.21-0.35)$

Trimethylamine N-oxide

$10.2(5.8-18.7)$

Trimethyllysine

\section{Endpoints}

In-hospital death

Death at 30 days

Death at 1 year

Death/HF at 30 days

Death/HF at 1 year

2 Data are reported as median (interquartile range) for continuous variables and as a $\%$ for

3 categorical.

4 BP, blood pressure; COPD, chronic obstructive pulmonary disease; eGFR, estimated

5 glomerular filtration rate; $\mathrm{HF}$, heart failure; $\mathrm{HFrEF}$, heart failure with reduced ejection

6 fraction $(<40 \%)$; HTN, hypertension; IHD, ischemic heart disease; JVP, jugular venous

7 pressure; NT-proBNP, N-terminal pro B-type natriuretic peptide; ADHERE; Acute

8 Decompensated Heart Failure National Registry; CI, confidence interval; GWTG-HF, Get

9 With The Guidelines ${ }^{\circledR}-H e a r t$ Failure, NRI, net reclassification index; OPTIMIZE-HF,

10 Organized Program to Initiate Lifesaving Treatment in Hospitalized Patients with Heart

11 Failure; NYHA, New York Heart Association.

*597 patients only 
1 Table 2 Multivariate analysis for death and death/HF

2

\section{Death}

30 days 1 year

\begin{tabular}{|c|c|c|c|c|c|c|}
\hline & HR & $95 \% \mathrm{CI}$ & p Value & HR & $95 \% \mathrm{CI}$ & p Value \\
\hline Acetyl-L-carnitine & 1.81 & $1.36-2.42$ & $<0.001$ & 1.33 & $1.14-1.54$ & $<0.001$ \\
\hline Betaine & 1.31 & $0.96-1.78$ & 0.086 & 1.13 & $0.96-1.34$ & 0.150 \\
\hline Choline & 1.07 & $0.81-1.41$ & 0.648 & 1.12 & $0.96-1.30$ & 0.165 \\
\hline$\gamma$-butyrobetaine & 1.59 & $1.17-2.17$ & 0.003 & 1.34 & $1.13-1.58$ & 0.001 \\
\hline L-carnitine & 1.63 & $1.25-2.11$ & $<0.001$ & 1.31 & $1.14-1.51$ & $<0.001$ \\
\hline TMA & 1.03 & $0.75-1.39$ & 0.874 & 1.08 & $0.93-1.25$ & 0.305 \\
\hline TMAO & 1.39 & $1.05-1.84$ & 0.022 & & $1.08-1.47$ & 0.004 \\
\hline TML & 1.40 & $1.07-1.85$ & 0.015 & 1.31 & $1.12-1.52$ & 0.001 \\
\hline
\end{tabular}

3

\section{Death/HF}

30 days 1 year

\begin{tabular}{ccccccc}
\hline & HR & $\mathbf{9 5 \%} \mathbf{C I}$ & p Value & HR & $\mathbf{9 5 \%} \mathbf{C I}$ & p Value \\
\hline Acetyl-L-carnitine & 1.49 & $1.19-1.88$ & 0.001 & 1.15 & $1.02-1.31$ & 0.026 \\
Betaine & 1.24 & $0.97-1.58$ & 0.093 & 1.08 & $0.95-1.23$ & 0.264 \\
Choline & 1.14 & $0.91-1.42$ & 0.254 & 1.06 & $0.94-1.21$ & 0.324 \\
$\gamma$-butyrobetaine & 1.45 & $1.13-1.85$ & 0.003 & 1.17 & $1.02-1.33$ & 0.023 \\
L-carnitine & 1.43 & $1.15-1.78$ & 0.001 & 1.16 & $1.03-1.31$ & 0.015 \\
TMA & 0.93 & $0.72-1.21$ & 0.592 & 1.03 & $0.91-1.17$ & 0.619 \\
TMAO & 1.38 & $1.10-1.73$ & 0.006 & 1.25 & $1.09-1.42$ & 0.001 \\
TML & 1.30 & $1.04-1.63$ & 0.021 & 1.19 & $1.05-1.35$ & 0.006 \\
\hline
\end{tabular}

5 Models were adjusted for sex, age, previous medical history (HF, ischaemic heart disease,

6 hypertension, and diabetes mellitus), New York Heart Association (NYHA) class, smoking

7 status, oedema, atrial fibrillation, systolic blood pressure, diastolic blood pressure, heart rate,

8 haemoglobin, respiratory rate, blood sodium and $(\log )$ NT-proBNP.

$9 \mathrm{CI}$, confidence interval; HR, hazard ratio; TMA, trimethylamine; TMAO, trimethylamine N-

10 oxide; TML, trimethyllysine. 
1 Table 3 Receiver operator characteristics for metabolites at 30 days and 1-year

2 outcomes of death/HF and, in-patient mortality

\begin{tabular}{|c|c|c|c|c|c|c|c|c|c|}
\hline & \multicolumn{3}{|c|}{30 days } & \multicolumn{3}{|c|}{1 year } & \multicolumn{3}{|c|}{ In-patent mortality } \\
\hline & AUC & $95 \% \mathrm{CI}$ & p Value & AUC & $95 \% \mathrm{CI}$ & p Value & $\mathbf{A U C}$ & $95 \% \mathrm{CI}$ & p Value \\
\hline Acetyl-L-carnitine & 0.675 & $0.621-0.730$ & $<0.001$ & 0.575 & $0.535-0.616$ & $<0.001$ & 0.744 & $0.673-0.816$ & $<0.001$ \\
\hline Betaine & 0.543 & $0.483-0.603$ & 0.156 & 0.535 & $0.494-0.576$ & 0.092 & 0.586 & $0.513-0.660$ & 0.022 \\
\hline Choline & 0.541 & $0.479-0.603$ & 0.193 & 0.525 & $0.483-0.566$ & 0.241 & 0.563 & $0.483-0.643$ & 0.121 \\
\hline$\gamma$-butyrobetaine & 0.627 & $0.568-0.686$ & $<0.001$ & 0.568 & $0.527-0.608$ & 0.001 & 0.680 & $0.604-0.756$ & $<0.001$ \\
\hline L-carnitine & 0.639 & $0.580-0.697$ & $<0.001$ & 0.563 & $0.523-0.604$ & 0.002 & 0.684 & $0.610-0.758$ & $<0.001$ \\
\hline TMA & 0.495 & $0.435-0.555$ & 0.865 & 0.538 & $0.497-0.579$ & 0.073 & 0.609 & $0.533-0.684$ & 0.005 \\
\hline TMAO & 0.636 & $0.579-0.693$ & $<0.001$ & 0.602 & $0.562-0.643$ & $<0.001$ & 0.658 & $0.581-0.736$ & $<0.001$ \\
\hline TML & 0.606 & $0.548-0.665$ & $<0.001$ & 0.568 & $0.528-0.609$ & 0.001 & 0.621 & $0.544-0.698$ & 0.002 \\
\hline
\end{tabular}

3

4 AUC, area under curve; CI, confidence interval; TMA, trimethylamine; TMAO,

5 trimethylamine $\mathrm{N}$-oxide; TML, trimethyllysine.

6

7

8 
1 Table $4 \quad$ Net reclassification index for in-patient mortality

\begin{tabular}{cccc}
\hline ADHERE & NRI & $\mathbf{9 5 \%}$ CI & p Value \\
\hline Acetyl-L-carnitine & 56.9 & $29.4-84.3$ & $<0.001^{*}$ \\
Betaine & 26.8 & $-0.7-54.3$ & 0.056 \\
Choline & 23.0 & $-4.4-50.4$ & 0.100 \\
$\gamma$-butyrobetaine & 39.3 & $11.8-66.8$ & $0.005^{*}$ \\
L-carnitine & 40.6 & $13.1-68.0$ & $0.004^{*}$ \\
TMA & 29.3 & $1.8-56.8$ & $0.037^{*}$ \\
TMAO & 18.4 & $-9.1-45.9$ & 0.189 \\
TML & 15.2 & $-12.3-42.7$ & 0.278 \\
\hline
\end{tabular}

2

\begin{tabular}{cccc}
\hline OPTIMIZE-HF & NRI & $\mathbf{9 5 \%}$ CI & p Value \\
\hline Acetyl-L-carnitine & 52.8 & $19.0-86.6$ & $0.002^{*}$ \\
Betaine & 25.6 & $-8.2-59.4$ & 0.138 \\
Choline & 36.3 & $2.5-70.1$ & $0.035^{*}$ \\
$\gamma$-butyrobetaine & 46.6 & $12.8-80.5$ & $0.007^{*}$ \\
L-carnitine & 37.8 & $4.0-71.6$ & $0.028^{*}$ \\
TMA & 16.9 & $-16.9-50.7$ & 0.326 \\
TMAO & 12.7 & $-21.1-46.5$ & 0.461 \\
TML & 10.0 & $-23.8-43.8$ & 0.561
\end{tabular}

3

\begin{tabular}{cccc}
\hline GWTG-HF & NRI & 95\% CI & p Value \\
\hline Acetyl-L-carnitine & 52.8 & $25.3-80.3$ & $<0.001^{*}$ \\
Betaine & 37.5 & $10.0-65.0$ & $0.007^{*}$ \\
Choline & 16.1 & $-11.4-43.6$ & 0.251 \\
$\gamma$-butyrobetaine & 38.0 & $10.5-65.5$ & $0.007^{*}$ \\
L-carnitine & 44.1 & $16.6-71.5$ & $0.002^{*}$ \\
TMA & 22.4 & $-5.0-49.9$ & 0.110 \\
TMAO & 25.9 & $-1.6-53.3$ & 0.065 \\
TML & 21.8 & $-5.7-49.3$ & 0.120 \\
\hline
\end{tabular}

4

5 ADHERE; Acute Decompensated Heart Failure National Registry; CI, confidence interval; 6 GWTG-HF, Get With The Guidelines ${ }^{\circledR}$-Heart Failure, NRI, net reclassification index; 7 OPTIMIZE-HF, Organized Program to Initiate Lifesaving Treatment in Hospitalized Patients 8 with Heart Failure; TMA, trimethylamine; TMAO, trimethylamine N-oxide; TML, 9 trimethyllysine. $* \mathrm{p}<0.05$ 


\section{FIGURES}

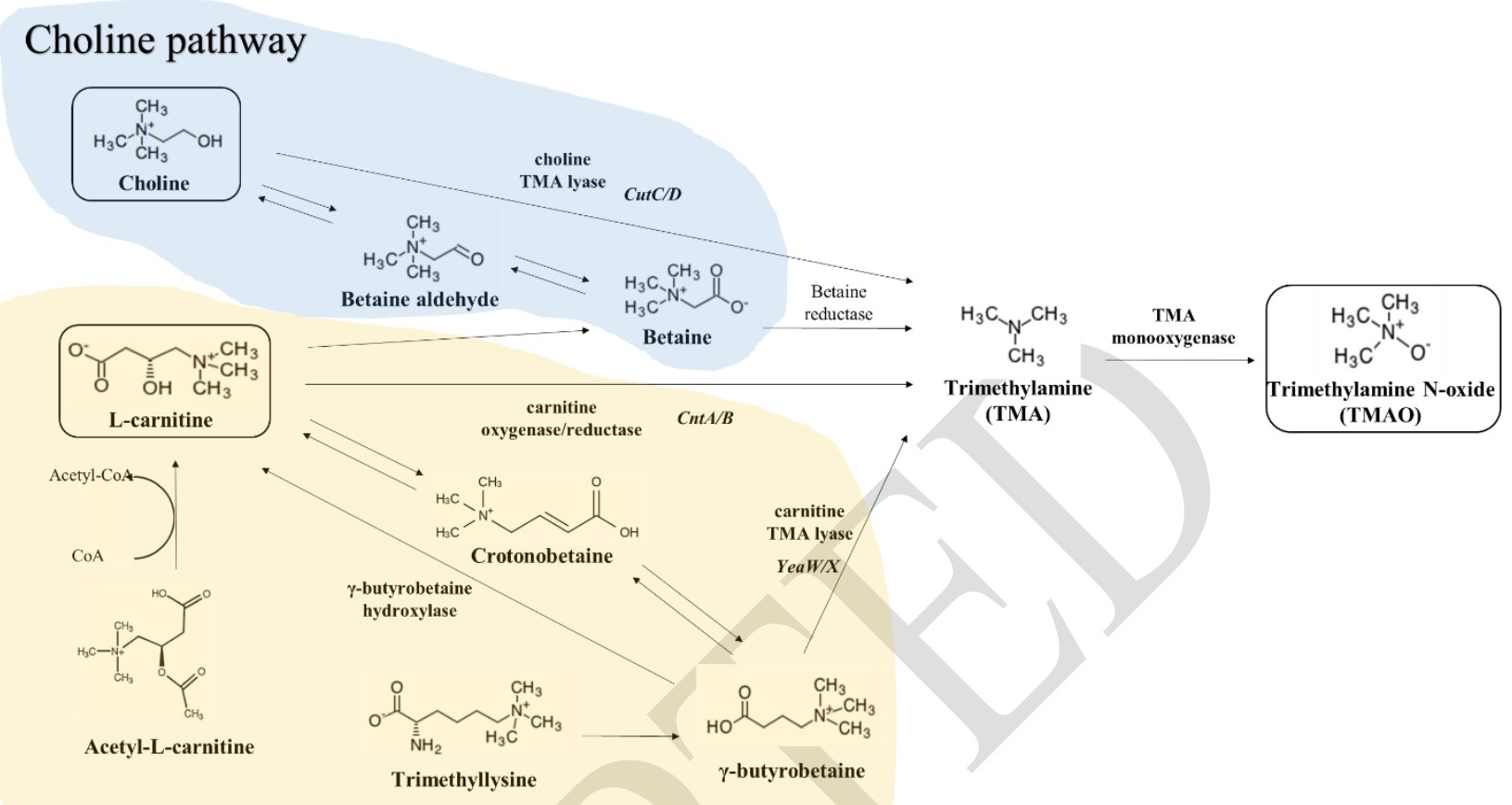

\section{Carnitine pathway}

4 Figure 1. Depiction of the TMAO-Choline/Carnitine metabolic pathway

5

6

7

8

9 

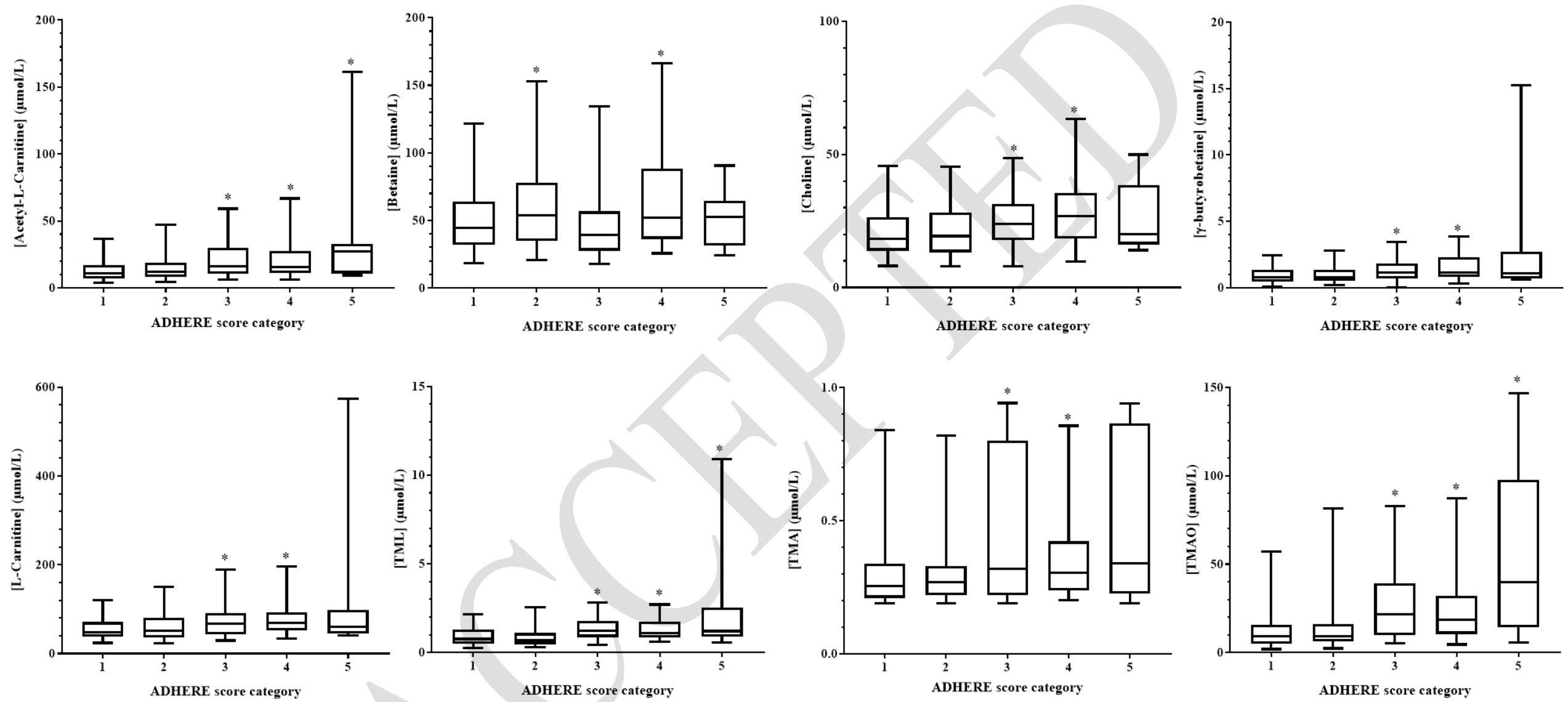

2

3 Figure 2. Box and whisker plot to show the distribution of gut-mediated metabolites in patients stratified by ADHERE score grouping.

4 ADHERE; Acute Decompensated Heart Failure National Registry. TML, trimethyllysine; TMA, trimethylamine; TMAO, trimethylamine N-oxide 

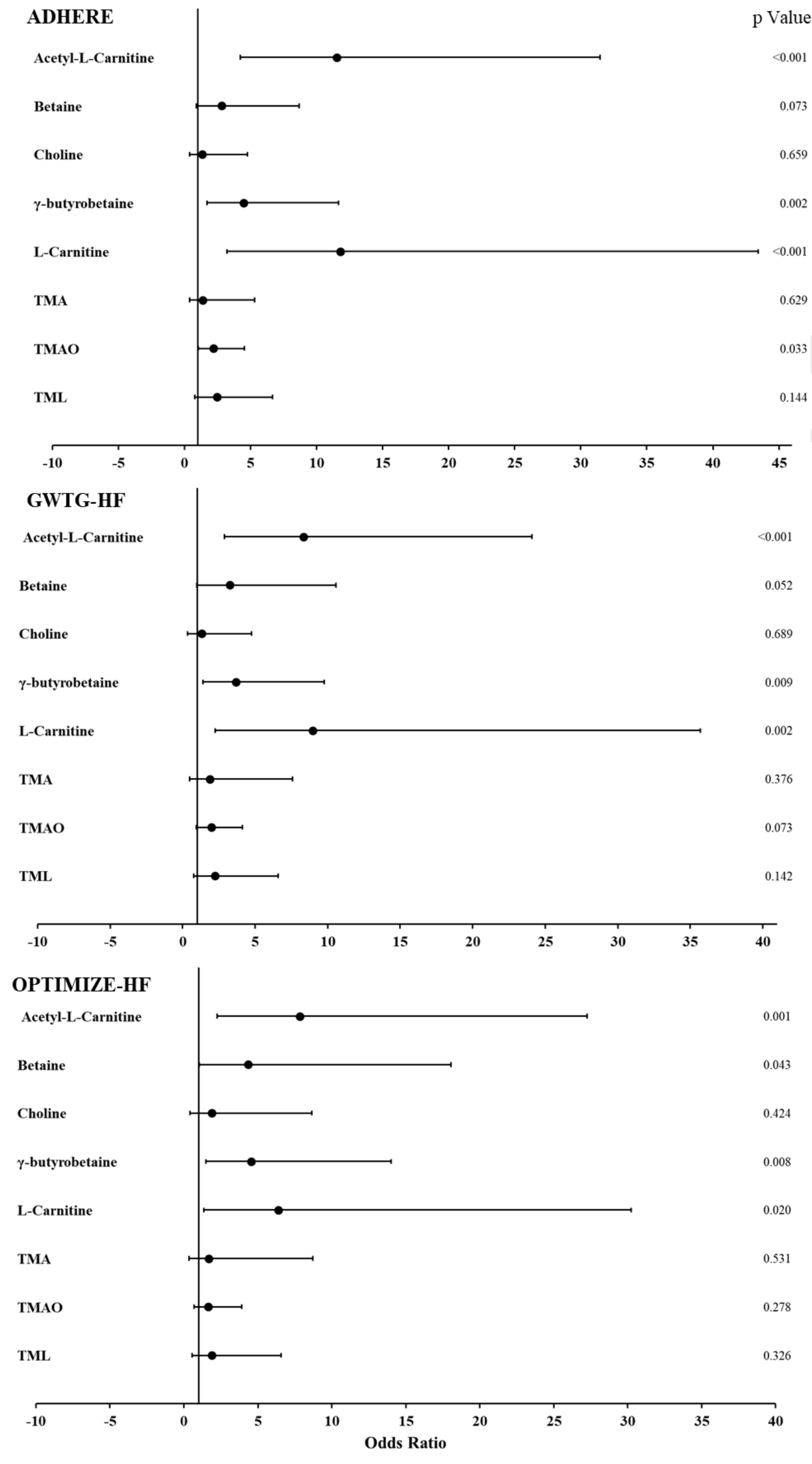
1 Figure 3. Forest plots to show binary logistic ORs (dots) and 95\% CIs (horizontal bars) for

2 cardiac risk factors for multivariate models for the individual components of in-hospital

3 mortality prediction algorithms of ADHERE, OPTIMIZE-HF and GWTG-HF registries with

4 the addition of metabolites.

5

6 TMA, trimethylamine; TMAO, trimethylamine N-oxide; TML, trimethyllysine; ADHERE;

7 Acute Decompensated Heart Failure National Registry; CI, confidence interval; GWTG-HF,

8 Get With The Guidelines ${ }^{\circledR}-$ Heart Failure, NRI, net reclassification index; OPTIMIZE-HF,

9 Organized Program to Initiate Lifesaving Treatment in Hospitalized Patients with Heart Failure

10 TML, trimethyllysine; TMA, trimethylamine; TMAO, trimethylamine N-oxide 
Muhammad Zubair Israr ${ }^{* 1}$, Dennis Bernieh ${ }^{* 1}$, Andrea Salzano ${ }^{* 2}$, Shabana Cassambai ${ }^{1}$, Yoshiyuki Yazaki ${ }^{1}$, Liam M Heaney ${ }^{1,3}$, Donald JL Jones ${ }^{1,4}$, Leong L Ng ${ }^{1}$, Toru Suzuki ${ }^{1}$

6 * contributed equally to this manuscript

Affiliations

${ }^{1}$ Department of Cardiovascular Sciences, University of Leicester and NIHR Leicester

Biomedical Research Centre, Leicester, UK

${ }^{2}$ IRCCS SDN, Diagnostic and Nuclear Research Institute, Naples, Italy

${ }^{3}$ School of Sport, Exercise \& Health Sciences, Loughborough University, Loughborough,

LE11 3TU, UK

${ }^{4}$ Department of Cancer Studies, University of Leicester, RKCSB, Leicester, LE2 7LX, UK

\section{Address for Correspondence:}

Prof Toru Suzuki

Department of Cardiovascular Sciences and NIHR Leicester Cardiovascular Biomedical 


\section{TABLE OF CONTENTS}

2 METHOD DEVELOPMENT AND VALIDATION

3 References

4 Supplementary Table 1. Retention times and MRM transitions for the metabolites and 5 internal standards

6 Supplementary Table 2. Linearity and sensitivity data for the ten metabolites in plasma

7 Supplementary Table 3. Intra- and inter-day accuracy and precision data for the ten

8 metabolites in plasma samples $(\mathrm{n}=6$ at all concentration levels)

9 Supplementary Table 4. Recovery and matrix effects data for the ten metabolites extracted from plasma samples at the low, medium and high QC concentration levels $(n=6)$

Supplementary Table 5. Effect of temperature and storage duration on metabolite concentrations measured from processed samples at the medium QC concentration range ... 16 Supplementary Figure 1. Overlaid extracted ion chromatogram from liquid chromatographytandem mass spectrometry with multiple reaction monitoring

Supplementary Table 6. Correlations between gut microbiota metabolites and associated clinical factors

Supplementary Table 7. Multivariate analysis for death and death/HF with renal marker influence

Supplementary Table 8 . Multivariate analysis for death/HF in ischaemic and non-ischaemic HF.

Supplementary Figure 2. Kaplan-Meier survival curve for outcomes of death and/or rehospitalization (death/HF) stratified by tertiles

Supplementary Figure 3. Kaplan-Meier survival curve for outcome of death stratified by rehospitalisation due to heart failure at (A) 30 days and (B) 1 year after combination of 


\section{Chemical and reagents}

4 Reference metabolite samples: O-acetyl-L-carnitine hydrochloride, betaine aldehyde chloride, betaine hydrochloride, choline chloride, crotonobetaine hydrochloride, gamma butyrobetaine hydrochloride, L-carnitine hydrochloride, trimethylamine hydrochloride,

TMAO (98.9\% purity), trimethyllysine hydrochloride were all purchased from Sigma Aldrich UK. Acetyl- $\mathrm{d}_{3}-\mathrm{L}$-carnitine hydrochloride, betaine - $\left(\mathrm{d}_{11}, 98 \%\right)$, choline chloride (trimethyl$\left.\mathrm{d}_{9}, 98 \%\right)$, L-carnitine-(trimethyl - $\left.\mathrm{d}_{9}, 98 \%\right)$, d9-TMAO ( $\geq 98 \%$ purity, $99.9 \%$ enrichment), trimethylamine DCL (TMA-d ${ }_{10}, 98 \%$ ) were purchased from Cambridge Isotopes USA. Formic acid, water, acetonitrile and methanol all Optima ${ }^{\mathrm{TM}} \mathrm{LC} / \mathrm{MS}$ Grade were obtained from Fisher Scientific UK. Ammonium hydroxide, extra pure, 25\% solution in water was purchased from ACROS Organics ${ }^{\mathrm{TM}}$. Stripped plasma $(4 \times$ charcoal, EDTA gender pooled: product code HMPLEDTA2STRPD-HEV-53432) was purchased from Seralab (Haywards Heath, UK).

\section{Preparation of calibrators and quality control samples}

Stock solutions for calibrators and QC samples of each metabolite were prepared separately. Standard stock solutions of each of the 10 metabolites were prepared in ultrapure water at a concentration of $5 \mathrm{mmol} / \mathrm{L}$. For betaine aldehyde, crotonobetaine, $\gamma$-BB and TML, no deuterated standards were commercially available, therefore betaine- $\mathrm{d}_{11}$ was used as the internal standard (I.S.) for quantitation of betaine aldehyde, crotonobetaine and $\gamma$-BB with TMAO-d9 successfully used for quantitation of TML. Stock solutions of the six internal standards were prepared in methanol at a concentration of $1 \mathrm{mmol} / \mathrm{L}$. The internal standard working solution was prepared at a final concentration of $10 \mu \mathrm{mol} / \mathrm{L}$ for acetyl-L-carnitine $\mathrm{d}_{3}$, 
1 betaine- $\mathrm{d}_{11}$, choline- $\mathrm{d}_{9}$, L-carnitine- $\mathrm{d}_{9}$, TMA- $\mathrm{d}_{10}$, and TMAO- $\mathrm{d}_{9}$, before it was stored at -

$280^{\circ} \mathrm{C}$ in aliquots.

Multicomponent working solutions for each metabolite were prepared freshly by appropriately diluting the stock solutions with water. The calibration ranges were chosen to cover the concentration ranges for the ten metabolites. A minimum of 8-point calibration curve was prepared by spiking charcoal stripped plasma with the correct concentration of multicomponent solution. These metabolites are endogenous compounds and are naturally present in human plasma, hence, it is difficult to find an analyte free matrix. It has been reported that in the absence of an analyte free matrix, an appropriate surrogate matrix $(1,2)$ such as pure water $(3,4)$, bovine serum albumin (5) and charcoal stripped plasma (6), can be used. Quality control (QC) samples at four concentration levels were prepared separately by spiking charcoal stripped plasma samples with multicomponent solutions of the ten metabolites.

\section{Sample extraction and derivatization}

Sample preparation involves a derivatization step where $20 \mu \mathrm{L}$ of methanol containing I.S. was added to $50 \mu \mathrm{l}$ of calibrators, QC or patient plasma samples, followed by the addition of $75 \mu \mathrm{l}$ of $50 \mathrm{mmol} / \mathrm{L}$ tert-butyl bromoacetate in acetonitrile and $25 \mu \mathrm{L}$ of $25 \%$ ammonium hydroxide. Samples were incubated at room temperature for 30 minutes, followed by addition of $50 \mu \mathrm{L}$ of acetonitrile containing $1 \%$ formic acid to each extract. The extract was vortexed for one minute and centrifuged at $16,900 \mathrm{rpm}$ for 20 minutes. The supernatant was collected into LC vials and analysed by LC-MS/MS. Derivatization was necessary due to TMA's low MS sensitivity and low blood levels (4). 
2 Analyses were achieved on a Shimadzu Nexera X2 LC-30AD online to a Shimadzu 8050

3 triple quadrupole mass spectrometer (TQ-MS). Separation of the ten metabolites and IS was

4 performed using an Acquity $\operatorname{UPLC}^{\circledR}$ BEH HILIC $(130 \AA, 100 \mathrm{~mm} \times 2.1 \mathrm{~mm}, 1.7 \mu \mathrm{m}$ pore size

5 column, Waters Corporation, USA) which was preceded by an Acquity UPLC ${ }^{\circledR}$ BEH HILIC

$6 \quad$ VanGuard pre-column (130 ̊, $1.7 \mu \mathrm{m}, 2.1 \times 5 \mathrm{~mm}$, Waters Corporation, USA). Separation

7 was performed using gradient elution mobile phase composed of a binary solvent mixture comprising of water containing $0.1 \%(\mathrm{v} / \mathrm{v})$ formic acid and $0.025 \%(\mathrm{v} / \mathrm{v})$ ammonium hydroxide (eluent A) and acetonitrile (eluent B). The gradient started with 95\% solvent B for 3 mins and was dropped to $60 \%$ solvent B over 1.5 mins, where it was maintained for 1.5 mins before ramping back to $95 \%$ in 8 mins. It was held at $95 \%$ solvent B for 2 mins before initiating the next injection. Chromatography was performed at $50{ }^{\circ} \mathrm{C}$ with a flow rate of 0.6 $\mathrm{mL} / \mathrm{min}$ and a run time of $10 \mathrm{~min}$. The autosampler was maintained at $4^{\circ} \mathrm{C}$ with an injection volume of $0.5 \mu \mathrm{L}$ for each sample. MS/MS acquisition was performed in multiple reaction monitoring (MRM) mode using electrospray positive ionization for detection. The optimum MS source and chamber conditions were as follows. Nebuliser gas flow: $3 \mathrm{~L} / \mathrm{min}$; heating gas flow: $10 \mathrm{~L} / \mathrm{min}$; drying gas flow: $10 \mathrm{~L} / \mathrm{min}$; interface temperature: $300{ }^{\circ} \mathrm{C}$; desolvation line (DL) temperature: 300 ${ }^{\circ} \mathrm{C}$; heat block temperature: $400{ }^{\circ} \mathrm{C}$; ion gauge (IG) vacuum: $2.0 \mathrm{E}-003 \mathrm{~Pa}$; pirani gauge $(\mathrm{PG})$ vacuum: $1.5 \mathrm{E}+002 \mathrm{~Pa}$ and CID gas: 270KPa. Detection of the ten metabolites and IS were achieved by monitoring the precursor to product ion transitions of each metabolite (Supplementary Table 1). Data acquisition and quantitative processing were accomplished using Labsolutions Software version 5.93 and Labsolutions Insight LCMS version 3.2 


\section{Method validation}

2 For the purposes of validation studies, four concentrations were chosen for the separate

3 preparation of quality control samples (QCs) at the LLOQ, low, medium and high

4 concentration levels for the ten metabolites and run alongside calibrators. To demonstrate that

5 the developed assay was fit for purpose, validation was conducted based upon international

6 guidelines (7). The selectivity, linearity, sensitivity (lower limit of quantitation (LLOQ)),

7 imprecision (intra and inter-assay accuracy and precision), matrix effects and stability were

8 determined for acetyl-L-carnitine, betaine, betaine aldehyde, choline, crotonobetaine, $\gamma$-BB,

9 L-carnitine, TMA, TMAO, and TML.

10 Linearity

11 Linearity was determined using a linear regression model. Calibrators of the ten metabolites were prepared in charcoal stripped plasma matrix and used to generate standard curves over the following concentration ranges: $0.1-100 \mu \mathrm{mol} / \mathrm{L}$ for acetyl-L-carnitine, crotonobetaine, $\gamma$-BB, TMA and TML; $0.5-200 \mu \mathrm{mol} / \mathrm{L}$ for betaine, choline, L-carnitine, and TMAO; $1.0-$ $400 \mu \mathrm{mol} / \mathrm{L}$ for betaine aldehyde. The concentrations of the ten metabolites in the plasma sample was determined from each metabolite/IS peak area ratio against nominal analyte concentration and a $1 / \mathrm{x}^{2}$ weighted linear regression was applied. The LLOQ was calculated based on a response $\geq$ five times the response of the zero calibrator with a signal to noise ratio of $10(7)$.

For the investigated dynamic range for each of the ten metabolites in plasma, $\mathrm{r}^{2}>0.99$ was obtained. The data (slope, intercept and mean correlation coefficient $\left.\left(\mathrm{r}^{2}\right)\right)$ for each metabolite is presented in Supplementary Table 2. The LLOQ with a signal to noise ratio of $\geq 10$ and the required assay precision and accuracy was $0.1 \mu \mathrm{mol} / \mathrm{L}$ for acetyl-L-carnitine, crotonobetaine, 
$1 \gamma$-BB, TMA and TML; $0.5 \mu \mathrm{mol} / \mathrm{L}$ for betaine, choline, L-carnitine, and TMAO; $1.0 \mu \mathrm{mol} / \mathrm{L}$

2 for betaine aldehyde.

\section{Assay imprecision (accuracy and precision)}

$4 \quad$ Replicate $(n=6)$ analyses of $(\mathrm{QC})$ samples, prepared at the LLOQ, low, medium and high

5 concentration levels of the ten metabolites were analysed to evaluate the inter- and intra-day

6 accuracy and precision. This involved three daily runs over a six-day period. Accuracy was

7 expressed as the relative error (\%RE) and precision as the relative standard deviation

8 (\%RSD). In line with FDA guidelines, a RE and RSD of $\leq 20 \%$ at the LLOQ and $\leq 15 \%$ at

9 other tested concentrations were considered acceptable.

10 Data obtained for within and between run variations for both accuracy and precision were

11 within the defined $\leq 20 \%$ limit at the LLOQ and $\leq 15 \%$ limit at all other concentrations in each run for the 10 metabolites. A summary of the results is presented in Supplementary Table 3.

\section{Carryover and selectivity}

Carryover was assessed by analysing blank samples consisting of methanol injections interspersed between QC samples and calibrators. Selectivity was achieved by using the most abundant product ion of each metabolite and IS for quantitation (Supplementary Table 1).

EICs from blank methanol injections interspersed between QC samples and calibrators showed that there was no carryover effect. Analysis of samples revealed there were no interfering endogenous compounds in plasma that coeluted with the quantitation of the ten metabolites when monitoring the selected product ions for the 10 metabolites (Supplementary Figure 1). 


\section{Recovery and matrix effect}

2 The efficiency of the extraction procedure was determined using replicate $(n=6)$ samples

3 prepared at the QC (low, medium and high) concentrations of the ten metabolites spiked into

4 plasma. Recovery was assessed by comparing the ratio of analyte to I.S. response from

5 plasma-spiked metabolite extracts, with those obtained from blank plasma extracts spiked

6 with metabolite solution standards of equal concentration.

Matrix effects were assessed with spiked plasma from blood samples obtained from 6 healthy volunteers. Replicate $(n=6)$ samples of the ten metabolites and I.S. spiked in plasma extracts to represent low, medium and high concentrations were prepared to evaluate ion suppression or enhancement. The prepared samples were compared with standards of equal concentration spiked into acetonitrile. The matrix effect was calculated using the formula $(\mathrm{B} / \mathrm{A}-1) \times 100$. Where A represents the ratio of the metabolite/I.S response from metabolite spiked into pure solvent and B represents the ratio of metabolite/I.S response from metabolite spiked into extracted plasma.

Recoveries for the ten metabolites were consistent and reproducible, with values between $70 \%$ and $100 \%$ for charcoal stripped and human plasma. The high extraction efficiencies indicate good extraction and metabolite stability under the applied extraction conditions. Recovery and matrix effect data for each metabolite at the QC concentrations investigated are summarised in Supplementary Table 4. There were no significant $(<20 \%)$ matrix effects on metabolite signal due to coeluents from plasma at all concentrations tested for each metabolite. These results demonstrated robustness of the extraction procedure and mechanism of ionisation for these metabolites. 


\section{Stability}

2 The stability of the 10 metabolites in plasma extracts was evaluated by analysing medium

3 QC samples by comparing the concentrations of the metabolites determined in samples

4 prepared freshly, with the same samples stored at $4^{\circ} \mathrm{C},-20^{\circ} \mathrm{C}$, and $-80^{\circ} \mathrm{C}$ after storage for 1,2

5 and 4 weeks. The freeze thaw samples were analysed with freshly prepared calibrators and

6 QC samples.

7 The ten metabolites were found to be stable when stored at temperatures of $4{ }^{\circ} \mathrm{C},-20{ }^{\circ} \mathrm{C}$ and -

$8 \quad 80{ }^{\circ} \mathrm{C}$ for up to 4 weeks in processed samples. No substantial changes in concentrations were

9 seen at the QC concentrations investigated for the 10 metabolites (Supplementary Table 5).

10 These findings indicate that, once samples are prepared, they can be stored in the fridge for 4

11 weeks or frozen to ensure stability over time. Additionally, pre-processed samples stored at temperatures of $4^{\circ} \mathrm{C}$ were stable for up to 16 weeks. 
2

3

4

5

6

7

8

9

\section{References}

1. Jones BR, Schultz GA, Eckstein JA, Ackermann BL. Surrogate matrix and surrogate analyte approaches for definitive quantitation of endogenous biomolecules. Bioanalysis 2012;4:2343-2356.

2. Wakamatsu A, Ochiai S, Suzuki E et al. Proposed selection strategy of surrogate matrix to quantify endogenous substances by Japan Bioanalysis Forum DG2015-15. Bioanalysis 2018;10:1349-1360.

3. Steuer C, Schütz P, Bernasconi L, Huber AR. Simultaneous determination of phosphatidylcholine-derived quaternary ammonium compounds by a LC-MS/MS method in human blood plasma, serum and urine samples. J Chromatogr B 2016;1008:206-211.

4. Zhao X, Zeisel SH, Zhang S. Rapid LC-MRM-MS assay for simultaneous quantification of choline, betaine, trimethylamine, trimethylamine $\mathrm{N}$-oxide, and creatinine in human plasma and urine. Electrophoresis 2015;36:2207-2214.

5. Liu J, Zhao M, Zhou J, Liu C, Zheng L, Yin Y. Simultaneous targeted analysis of trimethylamine-N-oxide, choline, betaine, and carnitine by high performance liquid chromatography tandem mass spectrometry. J Chromatogr B 2016;1035:42-48.

6. Heaney LM, Jones DJ, Mbasu RJ, Ng LL, Suzuki T. High mass accuracy assay for trimethylamine $\mathrm{N}$-oxide using stable-isotope dilution with liquid chromatography coupled to orthogonal acceleration time of flight mass spectrometry with multiple reaction monitoring. Anal Bioanal Chem 2016;408:797-804.

7. Administration USDoHaHSFaD. Bioanalytical Method Validation Guidance for Industry. 2018:41. 
1 Supplementary Table 1. Retention times and MRM transitions for the metabolites and

2 internal standards

\begin{tabular}{|c|c|c|c|c|}
\hline \multirow[b]{2}{*}{ Analyte } & \multirow[b]{2}{*}{$\begin{array}{c}\text { Retention time } \\
\text { (mins) }\end{array}$} & \multicolumn{2}{|c|}{ Transitions, $\mathbf{m} / \mathbf{z}$} & \multirow[b]{2}{*}{$\begin{array}{c}\text { Collision energy } \\
\text { (V) }\end{array}$} \\
\hline & & Precursor ion & Product ions & \\
\hline Acetyl-L-carnitine & 6.50 & 204.0 & $\mathbf{8 5 . 0 5 / 6 0 . 2 0}$ & -18 \\
\hline Betaine Aldehyde & 3.63 & 102.0 & $\mathbf{5 8 . 1 0} / 59.15$ & -23 \\
\hline Betaine & 5.13 & 118.0 & $\mathbf{5 8 . 1 0} / 59.15$ & -25 \\
\hline Choline & 4.16 & 104.1 & $58.10 / 60.15$ & -28 \\
\hline Crotonobetaine & 6.80 & 144.1 & $\mathbf{5 8 . 1 0} / 59.15$ & -25 \\
\hline$\gamma$-butyrobetaine & 6.74 & 146.1 & $87.05 / 64.10$ & -16 \\
\hline L-carnitine & 6.68 & 162.1 & $\mathbf{6 0 . 1 5} / 103.10$ & -16 \\
\hline TMA & 3.10 & 174.1 & $\mathbf{5 9 . 0 5} / 118.10$ & -24 \\
\hline TMAO & 6.42 & 76.2 & $\mathbf{5 8 . 1 0} / 59.15$ & -24 \\
\hline TML & 7.20 & 189.1 & 84.10/130.15 & -22 \\
\hline Acetyl-L-carnitine $-\mathrm{d}_{3}$ & 6.50 & 207.1 & 85.10/166.15 & -19 \\
\hline Betaine $-d_{11}$ & 5.13 & 129.3 & $66.25 / 58.10$ & -28 \\
\hline Choline $-\mathrm{d}_{9}$ & 4.16 & 113.1 & 66.10/69.20 & -32 \\
\hline L-carnitine $-\mathrm{d}_{9}$ & 6.68 & 171.1 & 69.20/103.20 & -18 \\
\hline TMAO $-\mathrm{d}_{9}$ & 6.41 & 85.2 & $\mathbf{6 6 . 1 5} / 68.20$ & -22 \\
\hline TMA $-\mathrm{d}_{10}$ & 3.10 & 183.1 & $\mathbf{6 6 . 0 5 / 1 2 7 . 1}$ & -39 \\
\hline
\end{tabular}

4

$5 \quad *$ The product ions in bold were used for quantitation

6 Reported collision energies are for product ions used for quantitation

$7 \mathrm{~m} / \mathrm{z}$, mass-to-charge ratio; TMA, trimethylamine; TMAO, trimethylamine N-oxide; TML,

8 trimethyllysine; $\mathrm{V}$, voltage 
1 Supplementary Table 2. Linearity and sensitivity data for the ten metabolites in plasma 2

\begin{tabular}{lccccc}
\hline Metabolite & Range $(\boldsymbol{\mu m o l} / \mathbf{L})$ & Slope & Intercept & $\mathbf{R}^{2}$ & LOQ $(\mu \mathrm{mol} / \mathbf{L})$ \\
\hline Acetyl-L-carnitine & $0.1-100$ & $0.352 \pm 0.024$ & $0.265 \pm 0.115$ & $0.998 \pm 0.0084$ & 0.1 \\
Betaine Aldehyde & $1.0-400$ & $0.156 \pm 0.013$ & $0.150 \pm 0.091$ & $0.997 \pm 0.0035$ & 1.0 \\
Betaine & $0.5-200$ & $0.562 \pm 0.101$ & $0.367 \pm 0.143$ & $0.994 \pm 0.0006$ & 0.5 \\
Choline & $0.5-200$ & $0.228 \pm 0.016$ & $0.127 \pm 0.096$ & $0.997 \pm 0.0074$ & 0.5 \\
Crotonobetaine & $0.1-100$ & $0.348 \pm 0.028$ & $0.315 \pm 0.052$ & $0.994 \pm 0.0034$ & 0.1 \\
$\gamma$-butyrobetaine & $0.1-100$ & $0.296 \pm 0.019$ & $0.760 \pm 0.125$ & $0.997 \pm 0.0015$ & 0.1 \\
L-carnitine & $0.5-200$ & $0.260 \pm 0.014$ & $0.185 \pm 0.080$ & $0.997 \pm 0.0032$ & 0.5 \\
TMA & $0.1-100$ & $0.072 \pm 0.002$ & $0.077 \pm 0.021$ & $0.999 \pm 0.0017$ & 0.1 \\
TMAO & $0.5-200$ & $0.114 \pm 0.005$ & $0.069 \pm 0.017$ & $0.997 \pm 0.0021$ & 0.5 \\
TML & $0.1-100$ & $0.123 \pm 0.016$ & $0.335 \pm 0.023$ & $0.997 \pm 0.0037$ & 0.1 \\
\hline
\end{tabular}

4 LOQ, limit of quantitation; $\mathrm{R}^{2}$, coefficient of determination; TMA, trimethylamine; TMAO, 5 trimethylamine $\mathrm{N}$-oxide; TML, trimethyllysine 
1 Supplementary Table 3. Intra- and inter-day accuracy and precision data for the ten

2 metabolites in plasma samples ( $\mathrm{n}=6$ at all concentration levels)

\begin{tabular}{|c|c|c|c|c|c|}
\hline \multirow[b]{2}{*}{ Metabolite } & \multirow[b]{2}{*}{$\begin{array}{l}\text { Nominal conc. } \\
(\mu \mathrm{mol} / \mathrm{L})\end{array}$} & \multicolumn{2}{|c|}{ Intra-day $^{\mathrm{a}}$} & \multicolumn{2}{|c|}{ Inter-day $^{\mathrm{b}}$} \\
\hline & & $\begin{array}{c}\text { Measured conc. } \\
(\mu \mathrm{mol} / \mathrm{L})\end{array}$ & RSD (\%) & $\begin{array}{l}\text { Measured conc. } \\
(\mu \mathrm{mol} / \mathrm{L})\end{array}$ & RSD (\%) \\
\hline \multirow[t]{4}{*}{ Acetyl-L-carnitine } & 0.1 & 0.12 & 6.25 & 0.11 & 4.65 \\
\hline & 0.3 & 0.35 & 8.70 & 0.36 & 0.94 \\
\hline & 5 & 5.04 & 0.85 & 5.85 & 1.72 \\
\hline & 50 & 50.14 & 4.71 & 49.03 & 2.00 \\
\hline \multirow[t]{4}{*}{ Betaine Aldehyde } & 1 & 1.06 & 7.29 & 1.07 & 1.19 \\
\hline & 3 & 3.02 & 4.87 & 3.08 & 1.91 \\
\hline & 25 & 25.04 & 6.24 & 25.92 & 1.80 \\
\hline & 200 & 195.55 & 1.36 & 196.29 & 0.49 \\
\hline \multirow[t]{4}{*}{ Betaine } & 0.5 & 0.51 & 7.26 & 0.51 & 1.32 \\
\hline & 1.5 & 1.49 & 4.16 & 1.52 & 1.72 \\
\hline & 25 & 24.73 & 12.92 & 24.86 & 0.55 \\
\hline & 100 & 94.39 & 7.92 & 98.45 & 3.65 \\
\hline \multirow[t]{4}{*}{ Choline } & 0.5 & 0.53 & 3.35 & 0.52 & 2.29 \\
\hline & 1.5 & 1.53 & 2.88 & 1.50 & 3.26 \\
\hline & 25 & 23.83 & 4.19 & 24.35 & 2.40 \\
\hline & 100 & 97.90 & 3.56 & 99.48 & 1.94 \\
\hline \multirow[t]{4}{*}{ Crotonobetaine } & 0.1 & 0.12 & 6.50 & 0.12 & 4.44 \\
\hline & 0.3 & 1.50 & 1.52 & 1.51 & 0.49 \\
\hline & 5 & 5.32 & 8.43 & 5.46 & 5.56 \\
\hline & 50 & 51.10 & 5.07 & 52.35 & 0.24 \\
\hline \multirow[t]{4}{*}{$\gamma$-butyrobetaine } & 0.1 & 0.12 & 6.14 & 0.13 & 2.28 \\
\hline & 0.3 & 0.31 & 2.80 & 0.32 & 0.54 \\
\hline & & 5.18 & 3.40 & 5.61 & 4.65 \\
\hline & 50 & 51.57 & 2.16 & 51.82 & 1.57 \\
\hline \multirow[t]{4}{*}{ L-carnitine } & 0.5 & 0.53 & 6.47 & 0.53 & 1.08 \\
\hline & 1.5 & 1.51 & 2.21 & 1.51 & 0.57 \\
\hline & 25 & 25.45 & 5.00 & 25.10 & 2.99 \\
\hline & 100 & 98.38 & 6.08 & 99.20 & 1.20 \\
\hline \multirow[t]{4}{*}{ TMA } & 0.1 & 0.13 & 9.08 & 0.13 & 2.19 \\
\hline & 0.3 & 0.31 & 3.11 & 0.32 & 0.38 \\
\hline & 5 & 5.27 & 0.49 & 5.51 & 4.37 \\
\hline & 50 & 51.57 & 4.73 & 52.33 & 0.61 \\
\hline \multirow[t]{4}{*}{ TMAO } & 0.5 & 0.55 & 5.45 & 0.53 & 4.42 \\
\hline & 1.5 & 1.56 & 1.92 & 1.53 & 1.54 \\
\hline & 25 & 24.17 & 1.77 & 24.18 & 2.43 \\
\hline & 100 & 96.05 & 3.17 & 99.45 & 3.00 \\
\hline \multirow[t]{4}{*}{ TML } & 0.1 & 0.10 & 6.76 & 0.12 & 2.92 \\
\hline & 0.3 & 0.32 & 2.03 & 0.32 & 1.00 \\
\hline & 5 & 5.47 & 3.22 & 5.32 & 3.36 \\
\hline & 50 & 53.52 & 4.64 & 51.40 & 1.85 \\
\hline
\end{tabular}


1 RSD, relative standard deviation; TMA, trimethylamine; TMAO, trimethylamine N-oxide;

2 TML, trimethyllysine

$3 \quad{ }^{a}$ Calculated for six replicates and three experiments conducted within a period of $24 \mathrm{~h}$

$4 \quad{ }^{b}$ Calculated for six replicates and three experiments conducted over a six day period 
1 Supplementary Table 4. Recovery and matrix effects data for the ten metabolites extracted

2 from plasma samples at the low, medium and high QC concentration levels $(n=6)$

\begin{tabular}{|c|c|c|c|c|c|}
\hline Metabolite & $\begin{array}{c}\text { Nominal } \\
\text { conc. }(\mu \mathrm{M})\end{array}$ & $\begin{array}{c}\text { Recovery } \\
(\%)\end{array}$ & $\begin{array}{l}\text { Standard } \\
\text { Deviation }\end{array}$ & $\begin{array}{c}\text { Mean Matrix } \\
\text { Effects (\%) }\end{array}$ & $\begin{array}{l}\text { Standard } \\
\text { Deviation } \\
\end{array}$ \\
\hline \multirow[t]{3}{*}{ Acetyl-L-carnitine } & 0.3 & 78.04 & 4.11 & 98.02 & 5.82 \\
\hline & 5 & 76.82 & 0.63 & 99.50 & 1.10 \\
\hline & 50 & 82.73 & 5.12 & 100.73 & 1.99 \\
\hline \multirow[t]{3}{*}{ Betaine Aldehyde } & 3 & 92.64 & 9.40 & 100.73 & 10.56 \\
\hline & 50 & 79.56 & 7.84 & 97.45 & 13.39 \\
\hline & 200 & 72.43 & 7.42 & 93.91 & 11.09 \\
\hline \multirow[t]{3}{*}{ Betaine } & 1.5 & 88.45 & 7.26 & 92.41 & 9.61 \\
\hline & 25 & 74.50 & 5.15 & 88.17 & 6.03 \\
\hline & 100 & 77.01 & 0.89 & 90.77 & 3.81 \\
\hline \multirow[t]{3}{*}{ Choline } & 1.5 & 87.11 & 10.58 & 102.76 & 7.71 \\
\hline & 25 & 86.78 & 2.32 & 93.14 & 4.17 \\
\hline & 100 & 87.69 & 1.33 & 101.13 & 4.94 \\
\hline \multirow[t]{3}{*}{ Crotonobetaine } & 0.3 & 82.01 & 5.95 & 90.33 & 5.36 \\
\hline & 5 & 75.71 & 3.47 & 98.70 & 12.12 \\
\hline & 50 & 81.80 & 2.42 & 102.33 & 6.29 \\
\hline \multirow[t]{3}{*}{$\gamma$-butyrobetaine } & 0.3 & 92.03 & 4.28 & 103.70 & 9.10 \\
\hline & 5 & 84.62 & 3.61 & 98.53 & 9.34 \\
\hline & 50 & 85.41 & 1.71 & 101.92 & 6.84 \\
\hline \multirow[t]{3}{*}{ L-carnitine } & 1.5 & 88.83 & 6.65 & 97.54 & 7.27 \\
\hline & 25 & 83.08 & 11.77 & 91.08 & 1.26 \\
\hline & 100 & 79.79 & 3.58 & 97.10 & 2.86 \\
\hline \multirow[t]{3}{*}{ TMA } & 0.3 & 102.83 & 7.47 & 89.58 & 5.66 \\
\hline & 5 & 105.85 & 5.69 & 85.19 & 7.34 \\
\hline & 50 & 99.10 & 3.03 & 84.61 & 4.62 \\
\hline \multirow[t]{2}{*}{ TMAO } & 1.5 & 78.61 & 2.32 & 101.83 & 4.82 \\
\hline & 25 & 87.66 & 0.69 & 95.11 & 1.52 \\
\hline & 100 & 88.68 & 1.71 & 99.41 & 2.12 \\
\hline \multirow[t]{3}{*}{ TML } & 0.3 & 91.51 & 8.07 & 84.40 & 4.30 \\
\hline & 5 & 92.07 & 1.60 & 84.45 & 6.03 \\
\hline & 50 & 91.46 & 3.50 & 97.50 & 7.12 \\
\hline
\end{tabular}

3

4 QC, quality control; TMA, trimethylamine; TMAO, trimethylamine N-oxide; TML, 5 trimethyllysine 
1 Supplementary Table 5. Effect of temperature and storage duration on metabolite

2 concentrations measured from processed samples at the medium QC concentration range

\begin{tabular}{|c|c|c|c|c|c|}
\hline Metabolite & $\begin{array}{c}\text { Storage } \\
\text { temperature } \\
\left({ }^{\circ} \mathrm{C}\right) \\
\end{array}$ & $\begin{array}{l}\text { Nominal } \\
\text { conc. } \\
(\mu \mathrm{M})\end{array}$ & $T=1$ week & $T=2$ weeks & $T=4$ weeks \\
\hline \multirow[t]{3}{*}{ Acetyl-L-carnitine } & 4 & 5 & $5.00 \pm 0.12$ & $5.32 \pm 0.17$ & $5.19 \pm 0.04$ \\
\hline & -20 & & $4.94 \pm 0.15$ & $5.12 \pm 0.05$ & $5.05 \pm 0.10$ \\
\hline & -80 & & $5.21 \pm 0.05$ & $5.08 \pm 0.11$ & $4.99 \pm 0.11$ \\
\hline \multirow[t]{3}{*}{ Betaine Aldehyde } & 4 & 50 & $48.25 \pm 1.05$ & $52.42 \pm 1.22$ & $52.73 \pm 0.92$ \\
\hline & -20 & & $47.98 \pm 2.96$ & $46.33 \pm 3.82$ & $48.10 \pm 2.76$ \\
\hline & -80 & & $50.89 \pm 3.88$ & $50.05 \pm 1.99$ & $49.88 \pm 3.55$ \\
\hline \multirow[t]{3}{*}{ Betaine } & 4 & 25 & $25.93 \pm 0.86$ & $25.20 \pm 0.78$ & $27.05 \pm 0.01$ \\
\hline & -20 & & $27.01 \pm 0.22$ & $26.88 \pm 1.30$ & $25.04 \pm 0.28$ \\
\hline & -80 & & $24.98 \pm 1.76$ & $26.32 \pm 0.84$ & $23.94 \pm 2.83$ \\
\hline \multirow[t]{3}{*}{ Choline } & 4 & 25 & $24.97 \pm 1.14$ & $24.72 \pm 0.49$ & $24.01 \pm 0.11$ \\
\hline & -20 & & $23.20 \pm 2.94$ & $22.93 \pm 1.66$ & $24.85 \pm 0.27$ \\
\hline & -80 & & $23.80 \pm 1.40$ & $25.80 \pm 0.86$ & $23.55 \pm 0.45$ \\
\hline \multirow[t]{3}{*}{ Crotonobetaine } & 4 & 5 & $5.22 \pm 0.02$ & $4.97 \pm 0.03$ & $5.10 \pm 0.03$ \\
\hline & -20 & & $5.03 \pm 0.05$ & $5.11 \pm 0.06$ & $5.05 \pm 0.20$ \\
\hline & -80 & & $4.79 \pm 0.15$ & $5.02 \pm 0.11$ & $4.83 \pm 0.55$ \\
\hline \multirow[t]{3}{*}{$\gamma$-butyrobetaine } & 4 & & $4.78 \pm 0.25$ & $4.84 \pm 0.08$ & $4.92 \pm 0.14$ \\
\hline & -20 & & $4.66 \pm 0.55$ & $5.51 \pm 0.12$ & $5.30 \pm 0.09$ \\
\hline & -80 & & $4.97 \pm 0.36$ & $5.02 \pm 0.44$ & $5.11 \pm 0.34$ \\
\hline \multirow[t]{3}{*}{ L-carnitine } & 4 & & $26.88 \pm 0.64$ & $26.37 \pm 0.71$ & $24.69 \pm 0.43$ \\
\hline & -20 & & $24.47 \pm 4.33$ & $23.97 \pm 2.56$ & $25.55 \pm 0.84$ \\
\hline & -80 & & $24.85 \pm 2.91$ & $24.86 \pm 3.85$ & $24.32 \pm 0.10$ \\
\hline \multirow[t]{3}{*}{ TMA } & 4 & 5 & $5.19 \pm 0.19$ & $4.97 \pm 0.04$ & $4.96 \pm 0.11$ \\
\hline & & & $5.06 \pm 0.14$ & $4.95 \pm 0.33$ & $5.06 \pm 0.50$ \\
\hline & -80 & & $4.93 \pm 0.22$ & $4.84 \pm 0.26$ & $5.08 \pm 0.04$ \\
\hline \multirow[t]{3}{*}{ TMAO } & 4 & 25 & $25.80 \pm 0.94$ & $24.65 \pm 0.04$ & $25.45 \pm 0.45$ \\
\hline & -20 & & $24.39 \pm 0.47$ & $25.05 \pm 0.65$ & $24.91 \pm 0.77$ \\
\hline & & & $27.11 \pm 0.39$ & $26.08 \pm 0.54$ & $23.15 \pm 0.54$ \\
\hline \multirow[t]{3}{*}{ TML } & 4 & 5 & $4.93 \pm 0.06$ & $5.33 \pm 0.06$ & $5.11 \pm 0.21$ \\
\hline & & & $5.22 \pm 0.32$ & $5.05 \pm 0.22$ & $4.99 \pm 0.16$ \\
\hline & -80 & & $5.10 \pm 0.40$ & $5.09+0.08$ & $5.06 \pm 0.42$ \\
\hline
\end{tabular}

3 QC, quality control; T, time; TMA, trimethylamine; TMAO, trimethylamine N-oxide; TML,

4 trimethyllysine 
1 Supplementary Figure 1. Overlaid extracted ion chromatogram from liquid

2 chromatography-tandem mass spectrometry with multiple reaction monitoring of 1)

3 trimethylamine, 2) betaine aldehyde, 3) choline, 4) betaine, 5) trimethylamine N-oxide, 6)

4 acetyl-L-carnitine, 7) L-carnitine, 8) $\gamma$-butyrobetaine, 9) crotonobetaine and 10)

5 trimethyllysine at the lower limit of quantitation

6

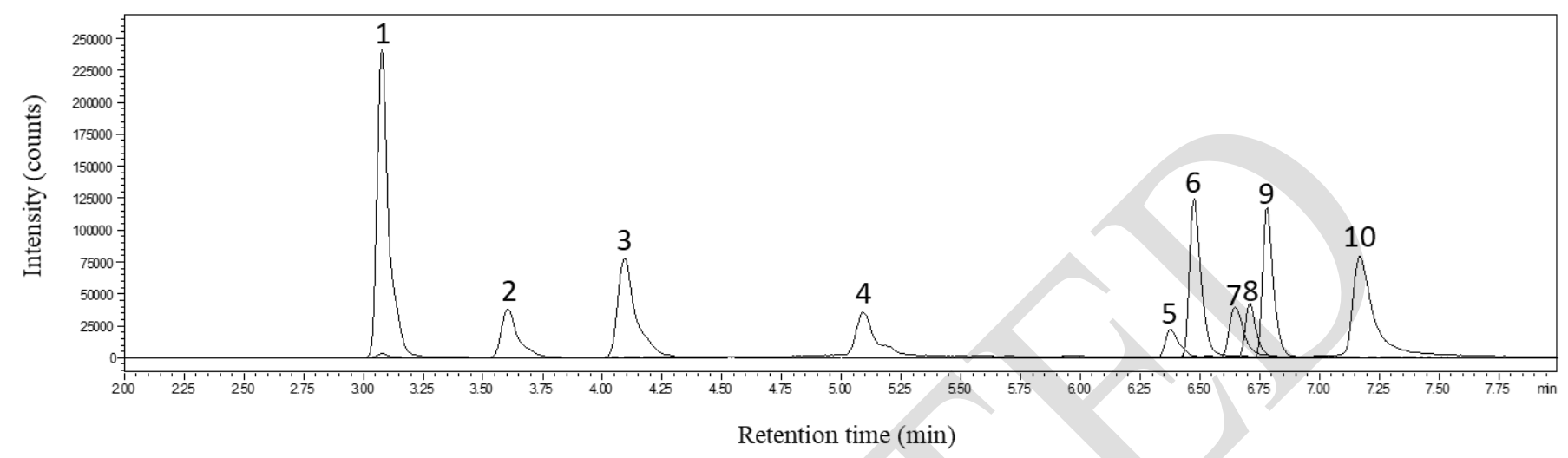

7 
Supplementary Table 6. Correlations between gut microbiota metabolites and associated clinical factors

\begin{tabular}{|c|c|c|c|c|c|c|c|c|c|c|c|c|c|c|c|c|}
\hline & \multicolumn{2}{|c|}{ Acetyl-L-carnitine } & \multicolumn{2}{|c|}{ Betaine } & \multicolumn{2}{|c|}{ Choline } & \multicolumn{2}{|c|}{$\gamma$-butyrobetaine } & \multicolumn{2}{|c|}{ L-carnitine } & \multicolumn{2}{|c|}{ TMA } & \multicolumn{2}{|c|}{ TMAO } & \multicolumn{2}{|c|}{ TML } \\
\hline & $r_{s}$ & p Value & $r_{s}$ & p Value & $r_{s}$ & p Value & $r_{s}$ & p Value & $r_{s}$ & p Value & $r_{s}$ & p Value & $\boldsymbol{r}_{s}$ & p Value & $\boldsymbol{r}_{s}$ & p Value \\
\hline eGFR & -0.242 & $<0.001$ & 0.008 & 0.816 & -0.215 & $<0.001$ & -0.208 & $<0.001$ & -0.162 & $<0.001$ & -0.123 & 0.001 & -0.437 & $<0.001$ & -0.313 & $<0.001$ \\
\hline Sodium & -0.213 & $<0.001$ & -0.089 & 0.014 & -0.031 & 0.401 & -0.124 & 0.001 & -0.197 & $<0.001$ & 0.011 & 0.772 & -0.004 & 0.909 & -0.019 & 0.603 \\
\hline Urea & 0.196 & $<0.001$ & 0.025 & 0.485 & 0.184 & $<0.001$ & 0.216 & $<0.001$ & 0.142 & $<0.001$ & 0.193 & $<0.001$ & 0.392 & $<0.001$ & 0.290 & $<0.001$ \\
\hline Systolic BP & -0.143 & $<0.001$ & -0.167 & $<0.001$ & -0.103 & 0.005 & -0.111 & 0.002 & -0.134 & $<0.001$ & -0.135 & $<0.001$ & -0.150 & $<0.001$ & -0.104 & 0.004 \\
\hline Potassium & 0.139 & $<0.001$ & -0.010 & 0.792 & 0.123 & 0.001 & 0.113 & 0.002 & 0.112 & 0.002 & 0.037 & 0.304 & 0.150 & $<0.001$ & 0.147 & $<0.001$ \\
\hline $\begin{array}{c}\text { Respiratory } \\
\text { Rate }\end{array}$ & 0.134 & $<0.001$ & -0.079 & 0.039 & -0.133 & 0.001 & 0.013 & 0.745 & 0.158 & 0.188 & -0.001 & 0.971 & -0.048 & 0.210 & 0.034 & 0.381 \\
\hline Diastolic BP & -0.118 & 0.001 & 0.008 & 0.829 & -0.032 & 0.379 & -0.036 & 0.323 & -0.082 & 0.026 & -0.051 & 0.166 & -0.136 & $<0.001$ & -0.093 & 0.011 \\
\hline Age & 0.104 & 0.003 & -0.168 & $<0.001$ & -0.017 & 0.628 & 0.080 & 0.023 & 0.014 & 0.701 & 0.006 & 0.861 & 0.177 & $<0.001$ & 0.043 & 0.222 \\
\hline NT-proBNP & 0.052 & 0.141 & 0.118 & 0.001 & 0.035 & 0.325 & 0.145 & $<0.001$ & -0.054 & 0.128 & 0.101 & 0.004 & 0.123 & $<0.001$ & 0.010 & 0.781 \\
\hline HR & -0.004 & 0.923 & 0.031 & 0.393 & -0.067 & 0.066 & -0.039 & 0.289 & -0.004 & 0.913 & 0.005 & 0.902 & -0.070 & 0.055 & -0.018 & 0.622 \\
\hline $\begin{array}{l}\text { Acetyl-L- } \\
\text { carnitine }\end{array}$ & & & 0.220 & $<0.001$ & 0.384 & $<0.001$ & 0.519 & $<0.001$ & 0.737 & $<0.001$ & 0.219 & $<0.001$ & 0.347 & $<0.001$ & 0.489 & $<0.001$ \\
\hline Betaine & & & & & 0.470 & $<0.001$ & 0.406 & $<0.001$ & 0.394 & $<0.001$ & 0.340 & $<0.001$ & 0.099 & 0.005 & 0.164 & $<0.001$ \\
\hline Choline & & & & & & & 0.505 & $<0.001$ & 0.510 & $<0.001$ & 0.355 & $<0.001$ & 0.381 & $<0.001$ & 0.476 & $<0.001$ \\
\hline $\begin{array}{c}\gamma- \\
\text { butyrobetaine }\end{array}$ & & & & & & & & & 0.590 & $<0.001$ & 0.229 & $<0.001$ & 0.324 & $<0.001$ & 0.645 & $<0.001$ \\
\hline L-Carnitine & & & & & & & & & & & 0.323 & $<0.001$ & 0.363 & $<0.001$ & 0.513 & $<0.001$ \\
\hline TMA & & & & & & & & & & & & & 0.306 & $<0.001$ & 0.235 & $<0.001$ \\
\hline TMAO & & & & & & & & & & & & & & & 0.475 & $<0.001$ \\
\hline TML & & & & & & & & & & & & & & & & \\
\hline
\end{tabular}

BP, blood pressure; eGFR, estimated glomerular filtration rate; HR, hazard ratio; NT-proBNP, N-terminal pro B-type natriuretic peptide; TMA,

trimethylamine; TMAO, trimethylamine $\mathrm{N}$-oxide; TML, trimethyllysine 
Supplementary Table 7. Multivariate analysis for death and death/HF with renal marker influence

\section{Death}

30 days 1 year

\begin{tabular}{ccccccc}
\hline & HR & 95\% CI & p Value & HR & 95\% CI & p Value \\
\hline Acetyl-L-carnitine & 1.37 & $1.01-1.85$ & $0.045^{*}$ & 1.15 & $0.98-1.36$ & 0.090 \\
Betaine & 1.24 & $0.92-1.69$ & 0.159 & 1.13 & $0.95-1.33$ & 0.166 \\
Choline & 0.82 & $0.61-1.10$ & 0.191 & 0.98 & $0.84-1.16$ & 0.843 \\
$\gamma$-butyrobetaine & 1.25 & $0.93-1.68$ & 0.146 & 1.19 & $1.01-1.40$ & $0.042^{*}$ \\
L-carnitine & 1.27 & $0.96-1.66$ & 0.091 & 1.16 & $1.00-1.35$ & 0.057 \\
TMA & 0.81 & $0.58-1.13$ & 0.209 & 1.00 & $0.86-1.17$ & 0.963 \\
TMAO & 0.93 & $0.69-1.26$ & 0.638 & 1.07 & $0.89-1.27$ & 0.469 \\
TML & 1.06 & $0.79-1.42$ & 0.708 & 1.14 & $0.97-1.34$ & 0.117 \\
\hline
\end{tabular}

\section{Death/HF}

\begin{tabular}{ccccccc} 
& \multicolumn{3}{c}{ 30 days } & \multicolumn{2}{c}{$\mathbf{1}$ year } \\
\hline & HR & $\mathbf{9 5 \%} \mathbf{C I}$ & $\mathbf{p}$ Value & HR & $\mathbf{9 5 \%}$ CI & p Value \\
\hline Acetyl-L-carnitine & 1.20 & $0.95-1.53$ & 0.131 & 1.02 & $0.89-1.17$ & 0.751 \\
Betaine & 1.19 & $0.93-1.52$ & 0.159 & 1.07 & $0.94-1.22$ & 0.325 \\
Choline & 0.94 & $0.75-1.18$ & 0.597 & 0.97 & $0.85-1.10$ & 0.619 \\
$\gamma$-butyrobetaine & 1.20 & $0.94-1.52$ & 0.139 & 1.06 & $0.93-1.23$ & 0.402 \\
L-carnitine & 1.18 & $0.94-1.48$ & 0.150 & 1.04 & $0.92-1.18$ & 0.502 \\
TMA & 0.79 & $0.60-1.03$ & 0.083 & 0.97 & $0.86-1.11$ & 0.684 \\
TMAO & 1.05 & $0.82-1.34$ & 0.688 & 1.11 & $0.96-1.28$ & 0.174 \\
TML & 1.04 & $0.82-1.32$ & 0.772 & 1.07 & $0.94-1.23$ & 0.325 \\
\hline
\end{tabular}

Adjusted for sex, age, PH (HF, IHD, HTN, DM), NYHA, smoker, oedema, atrial fibrillation, sBP, dBP, HR, Hb, respiratory rate, Na, (log)NT-proBNP, urea, eGFR; CI, confidence interval; HR, hazard ratio; TMA, trimethylamine; TMAO, trimethylamine N-oxide; TML, trimethyllysine. 
Supplementary Table 8. Multivariate analysis for death/HF in ischaemic and nonischaemic HF

\begin{tabular}{ccccc}
\hline 30 days & \multicolumn{2}{c}{ Ischaemic HF } & HR (95\% CI) & p Value \\
\hline Acetyl-L-carnitine & $1.38(0.89-2.14)$ & 0.146 & $1.49(1.11-1.99)$ & $0.008^{*}$ \\
Betaine & $1.28(0.86-1.91)$ & 0.230 & $1.10(0.79-1.54)$ & 0.563 \\
Choline & $0.89(0.59-1.35)$ & 0.591 & $1.21(0.92-1.58)$ & 0.180 \\
$\gamma$-butyrobetaine & $1.52(0.96-2.42)$ & 0.076 & $1.35(0.99-1.84)$ & 0.062 \\
L-carnitine & $1.14(0.77-1.68)$ & 0.527 & $1.55(1.16-2.09)$ & $0.003^{*}$ \\
TMA & $1.02(0.62-1.69)$ & 0.933 & $0.91(0.66-1.24)$ & 0.538 \\
TMAO & $1.04(0.66-1.64)$ & 0.858 & $1.48(1.12-1.95)$ & $0.006^{*}$ \\
TML & $1.36(0.85-2.16)$ & 0.200 & $1.24(0.95-1.62)$ & 0.119 \\
\hline 1 year & & & $1.11(0.93-1.32)$ & 0.235 \\
Acetyl-L-carnitine & $1.22(0.99-1.51)$ & 0.057 & $1.01(0.84-1.22)$ & 0.916 \\
Betaine & $1.11(0.91-1.36)$ & 0.302 & $1.07(0.91-1.26)$ & 0.394 \\
Choline & $1.03(0.84-1.25)$ & 0.800 & $1.23(1.04-1.47)$ & 0.019 \\
$\gamma$-butyrobetaine & $1.09(0.86-1.38)$ & 0.485 & $1.20(1.01-1.43)$ & $0.041^{*}$ \\
L-carnitine & $1.10(0.91-1.34)$ & 0.309 & $1.04(0.88-1.22)$ & 0.660 \\
TMA & $1.10(0.88-1.36)$ & 0.407 & $1.27(1.08-1.49)$ & $0.004^{*}$ \\
TMAO & $1.18(0.93-1.51)$ & 0.180 & $1.18(1.00-1.39)$ & $0.047^{*}$ \\
TML & $1.18(0.94-1.49)$ & 0.152 & &
\end{tabular}

Adjusted for sex, age, PH (HF, IHD, HTN, DM), NYHA, smoker, oedema, atrial fibrillation, sBP, dBP, HR, $\mathrm{Hb}$, respiratory rate, $\mathrm{Na},(\log ) \mathrm{NT}$-proBNP; CI, confidence interval; HR, hazard ratio; TMA, trimethylamine; TMAO, trimethylamine $\mathrm{N}$-oxide; TML, trimethyllysine. * $\mathrm{p}<0.05$. 
Supplementary Figure 2. Kaplan-Meier survival curve for outcomes of death and/or rehospitalization (death/HF) stratified by tertiles

$\mathbf{A}$

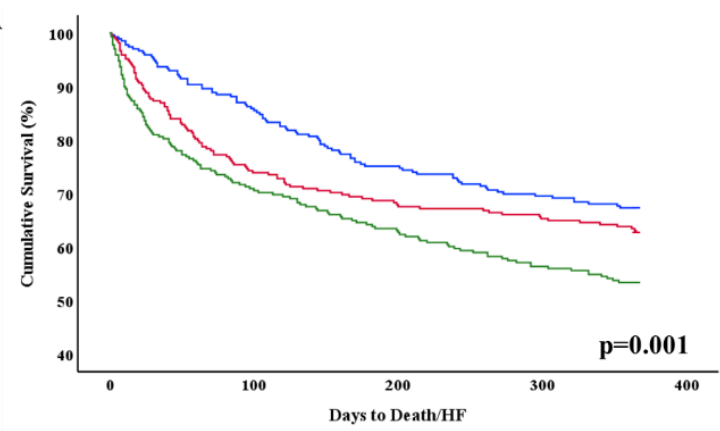

B

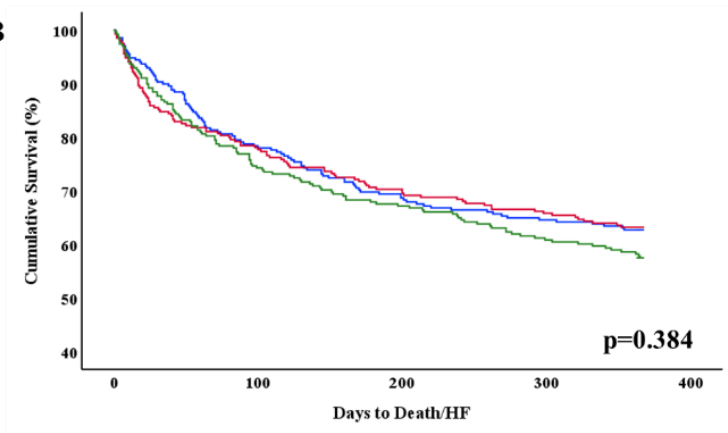

C
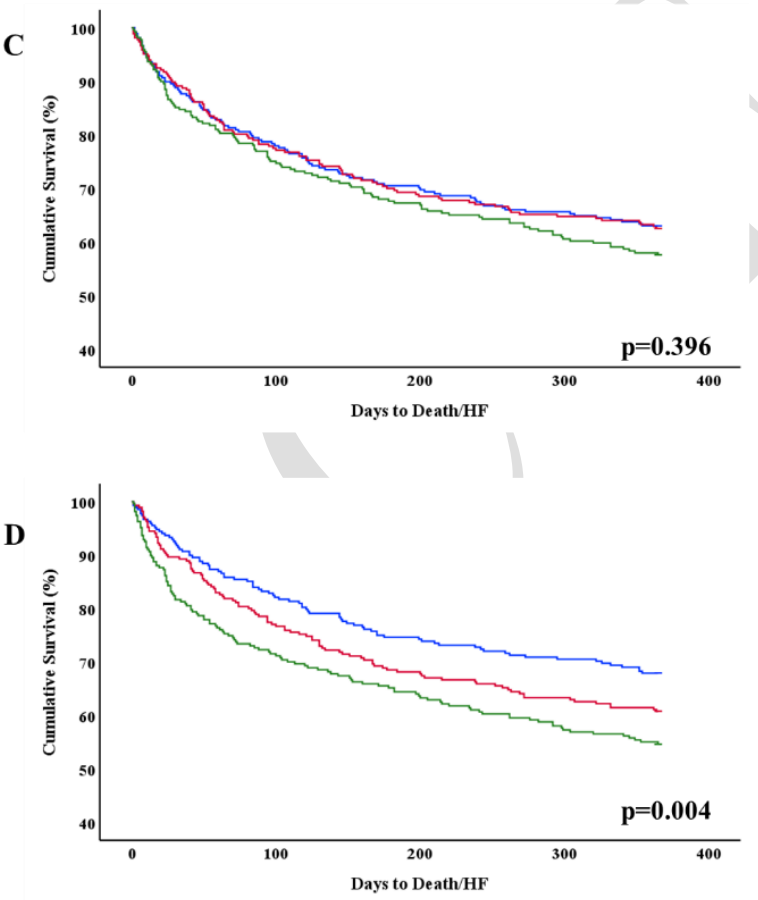

E

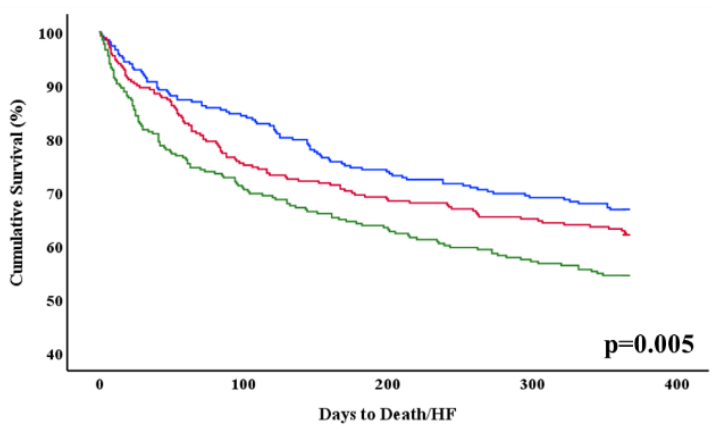

F

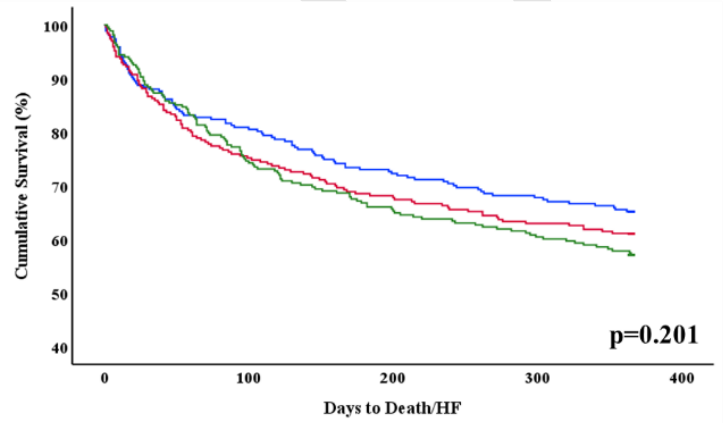

G
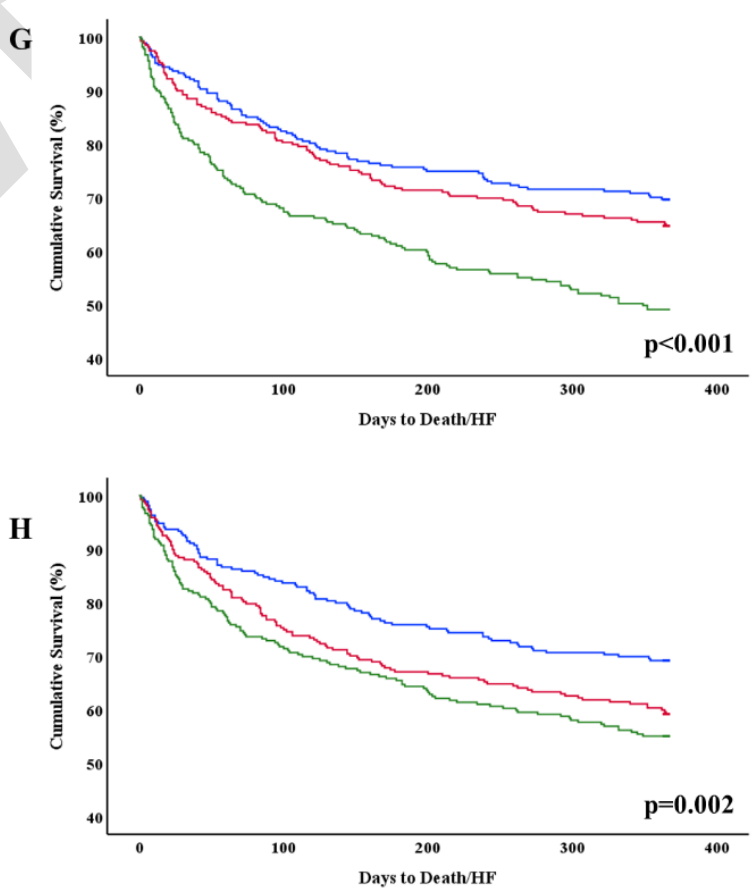

$\mathrm{T} 2$ T3

A- Acetyl-L-carnitine, B- Betaine, C- Choline, D- $\gamma$-butyrobetaine, E- L-carnitine, F- Trimethylamine, G-

Trimethylamine $\mathrm{N}$-oxide, $\mathrm{H}$ - Trimethyllysine 
Supplementary Figure 3. Kaplan-Meier survival curve for outcome of death stratified by tertiles
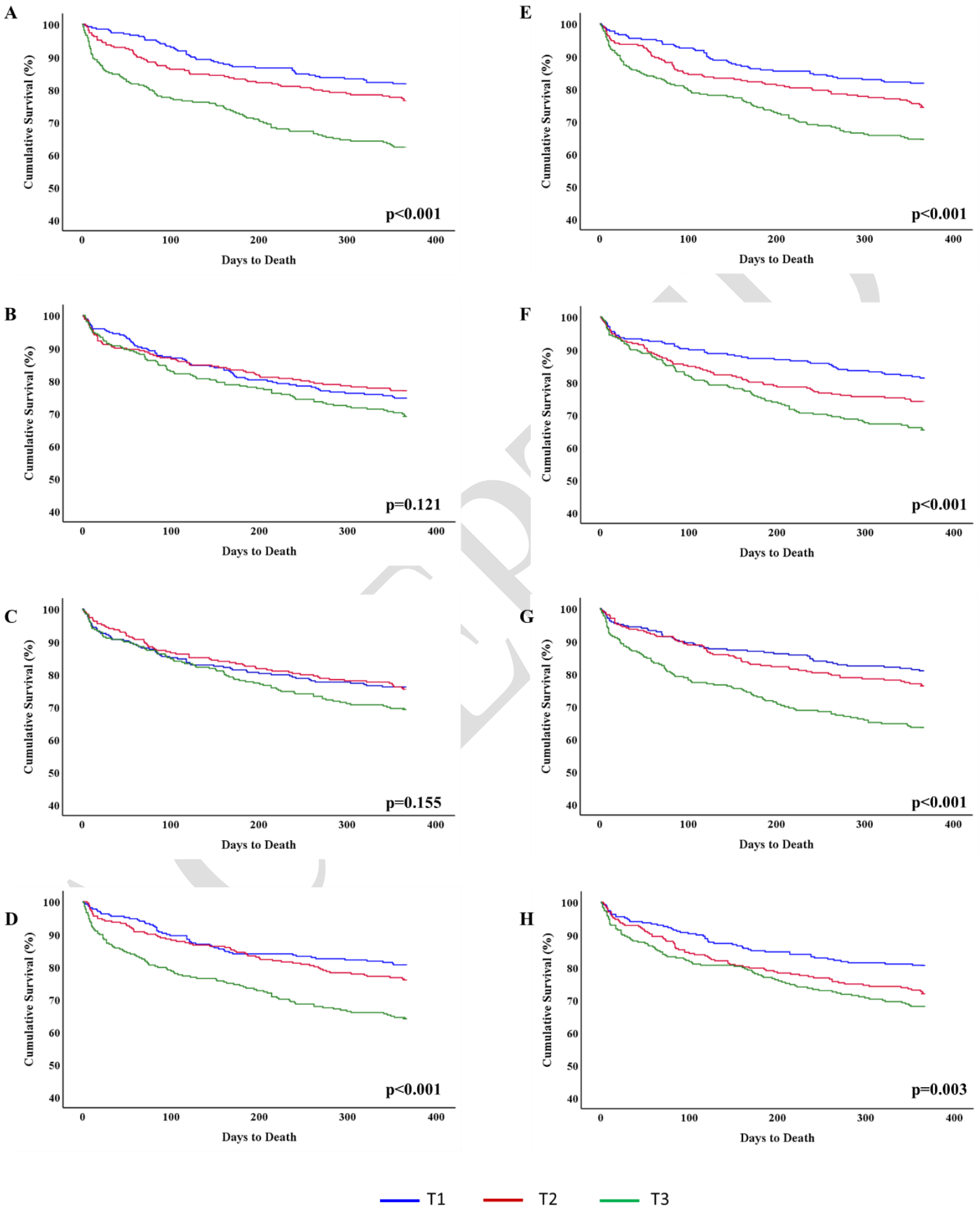

A- Acetyl-L-carnitine, B- Betaine, C- Choline, D- $\gamma$-butyrobetaine, E- L-carnitine, F- Trimethylamine, G-

Trimethylamine N-oxide, H- Trimethyllysine 
Supplementary Figure 4. Mantel-Cox survival analysis for all cause death and/or rehospitalisation due to heart failure at (A) 30 days and (B) 1 year after combination of TMAO and acetyl-L-carnitine

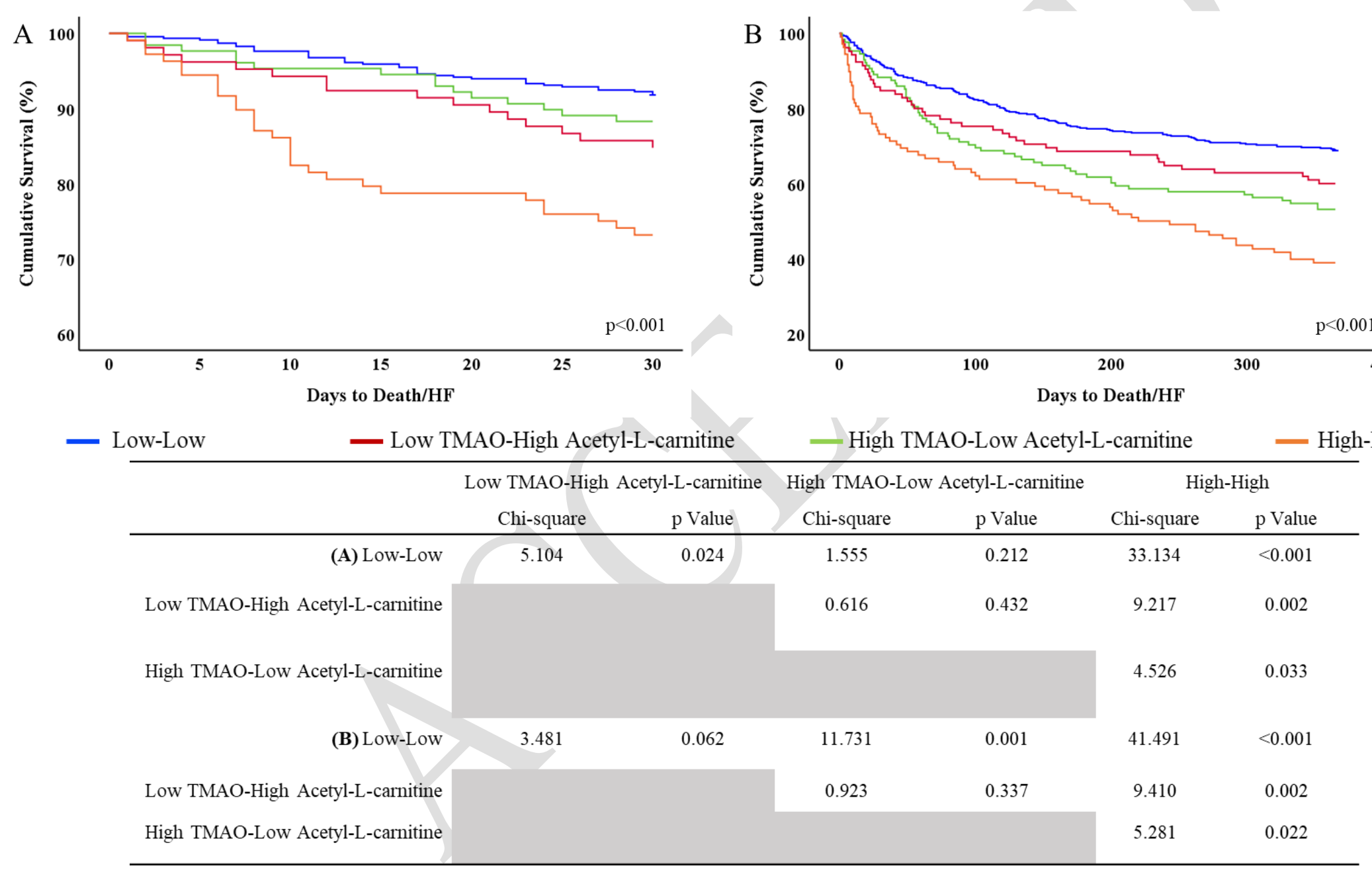

